J. Nonlinear Var. Anal. 3 (2019), No. 2, pp. 205-224

Available online at http://jnva.biemdas.com

https://doi.org/10.23952/jnva.3.2019.2.09

\title{
A PROJECTED SUBGRADIENT-PROXIMAL METHOD FOR SPLIT EQUALITY EQUILIBRIUM PROBLEMS OF PSEUDOMONOTONE BIFUNCTIONS IN BANACH SPACES
}

\author{
FERDINARD U. OGBUISI ${ }^{1,2}$, YEKINI SHEHU ${ }^{2, *}$ \\ ${ }^{1}$ School of Mathematics, Statistics and Computer Science, University of KwaZulu-Natal, Durban, South Africa \\ ${ }^{2}$ Department of Mathematics, University of Nigeria, Nsukka, Nigeria
}

\begin{abstract}
In this paper, we propose a simultaneous projected subgradient-proximal type iterative algorithm to solve a split equality equilibrium problem with pseudomonotone bifunctions in 2-uniformly convex and uniformly smooth Banach spaces. We obtain convergence results under some mild conditions on the bifunctions. Furthermore, we also give applications to the domain decomposition for PDEs.

Keywords. Projected subgradient-proximal method; Pseudomonotone bifunctions; Split equality equilibrium problem; 2uniformly convex Banach space; Uniformly smooth Banach space.
\end{abstract}

2010 Mathematics Subject Classification. 49J53, 65K10.

\section{INTRODUCTION}

Let $E_{1}, E_{2}$ and $E_{3}$ be Banach spaces. Let $C_{1}$ and $C_{2}$ be nonempty closed and convex subsets of $E_{1}$ and $E_{2}$, respectively. Let $f_{1}: C_{1} \times C_{1} \rightarrow \mathbb{R}$ and $f_{2}: C_{1} \times C_{2} \rightarrow \mathbb{R}$ be bifunctions. Let $A_{1}: E_{1} \rightarrow E_{3}$ and $A_{2}: E_{2} \rightarrow E_{3}$ be bounded linear operators. The split equality equilibrium problem (SEEP) is to find $x^{*} \in C_{1}$ and $y^{*} \in C_{2}$ such that

$$
f_{1}\left(x^{*}, x\right) \geq 0, \forall x \in C_{1}, f_{2}\left(y^{*}, y\right) \geq 0 \forall y \in C_{2},
$$

and

$$
A_{1} x^{*}=A_{2} y^{*}
$$

We denote by $S$ the solution set of SEEP (1.1)-(1.2).

Observe that if $E_{2}=E_{3}$ and $A_{2}$ is the identity mapping of $E_{2}$, then SEEP (1.1)-(1.2) is reduced to the following Split Equilibrium Problem (SEP) (see, [19, 25, 26]): find

$$
x^{*} \in C_{1} \text { such that } f_{1}\left(x^{*}, x\right) \geq 0, \forall x \in C_{1},
$$

and

$$
y^{*}=A x^{*} \in C_{2} \text { such that } f_{2}\left(y^{*}, y\right) \geq 0, \forall x \in C_{2} .
$$

${ }^{*}$ Corresponding author.

E-mail addresses: ferdinard.ogbuisi@unn.edu.ng (F.U. Ogbuisi), yekini.shehu@unn.edu.ng (Y. Shehu).

Received March 24, 2019; Accepted May 9, 2019.

(C)2019 Journal of Nonlinear and Variational Analysis 
If $f_{2}=0$ and $C_{2}=E_{2}$, then SEP (1.3) -(1.4) is reduced to the following Equilibrium Problem (EP) (see [9, 23]): find $x^{*} \in C$ such that

$$
f\left(x^{*}, y\right) \geq 0, \forall y \in C .
$$

Let us denote the set of solutions of EP (1.5) by $E P(C, f)$. EP (1.5) has been applied to various important problems such as physics, optimization and economics (see [27, 33]). If for $i=1,2$, we let $f_{i}(x, y)=\left\langle B_{i} x, y-x\right\rangle$, where $B_{i}: E_{i} \rightarrow E_{i}^{*}$ is an operator. Then SEEP (1.1)-(1.2) becomes the split equality variational inequality problems studied in [18]. Consequently, we have that SEEP (1.1)-(1.2) is also a generalization of the split variational inequality problem considered in [14]. Another special case of SEP (1.3) -(1.4) is the Split Feasibility Problem (SFP). The SFP was first considered in Euclidean spaces by Censor and Elfving [12] for modelling inverse problems which have applications in phase retrievals and medical image reconstruction. The SFP has been studied in more general frameworks including Hilbert spaces and Banach spaces; see $[13,28,29,30]$ and the references therein. The SFP has also been applied in image restoration, computer tomography and radiation therapy treatment planning; see [11, 13] and the references therein. Authors also considered some generalisations of the SFP such as the Split Common Fixed Point Problem (SCFPP) [15], Split Equality Fixed Point Problem (SEFPP) [12, 16], etc.

Recently, Gebrie and Wangkeeree [19] proposed a projected subgradient-proximal algorithm for solving the following Fixed Point-Set Constrained Split Equilibrium Problems (FPSCSEPs) in Hilbert spaces:

$$
\text { find } x^{*} \in C_{1} \text { such that }\left\{\begin{array}{l}
x^{*} \in F(T), \\
f_{1}\left(x^{*}, y\right) \geq 0, \forall y \in C_{1}, \\
u^{*}=A x^{*} \in F(V), \\
f_{2}\left(u^{*}, u\right) \geq 0, \forall u \in C_{2},
\end{array}\right.
$$

where $T: C_{1} \rightarrow C_{1}$ and $V: C_{2} \rightarrow C_{2}$ are nonexpansive mappings. They assumed that bifunctions $f_{2}$ : $C_{2} \times C_{2} \rightarrow \mathbb{R}$ and $f_{1}: C_{1} \times C_{1} \rightarrow \mathbb{R}$ satisfy the following Condition A and Condition B respectively.

\section{Condition A}

(A1) $f_{2}(u, u)=0$ for all $u \in C_{2}$.

(A2) $f_{2}$ is monotone on $C_{2}$, i.e., $f_{2}(u, v)+f_{2}(v, u) \leq 0$, for all $u, v \in C_{2}$.

(A3) For each $u, v, w \in C_{2}$,

$$
\underset{t \downarrow 0}{\limsup } f_{2}(t w+(1-t) u, v) \leq f_{2}(u, v)
$$

(A4) $f_{2}(u,$.$) is convex and lower semicontinuous on C_{2}$ for each $u \in C_{2}$.

\section{Condition B}

(B1) $f_{1}(x, x)=0$ for all $x \in C_{1}$.

(B2) $f_{1}$ is pseudomonotone on $C_{1}$ with respect to $x \in E P\left(f_{1}, C\right)$, i.e., if $x \in E P\left(f_{1}, C_{1}\right)$ then $f_{1}(x, y) \geq 0$ implies $f_{1}(y, x) \leq 0 \forall y \in C_{1}$.

(B3) $f_{1}$ satisfies the following condition, which called the strict paramonotonicity property:

$$
x \in E P\left(f_{1}, C_{1}\right), y \in C_{1}, f_{1}(y, x)=0 \Rightarrow y \in E P\left(f_{1}, C_{1}\right) .
$$

(B4) $f_{1}$ is jointly weakly upper semicontinuous on $C_{1} \times C_{1}$ in the sense that, if $x, y \in C_{1}$ and $\left\{x_{k}\right\},\left\{y_{k}\right\} \subset$ $C_{1}$ converge weakly to $x$ and $y$, respectively, then $f_{1}\left(x_{k}, y_{k}\right) \rightarrow f_{1}(x, y)$ as $k \rightarrow \infty$.

(B5) $f_{1}(x,$.$) is convex, lower semicontinuous and subdifferentiable on C_{1}$, for all $x \in C$. 
(B6) If $\left\{x_{k}\right\}$ is a bounded sequence in $C_{1}$ and $\varepsilon_{k} \rightarrow 0$, then the sequence $\left\{w_{k}\right\}$ with $w_{k} \in \partial_{\varepsilon_{k}} f_{1}\left(x_{k},.\right)\left(x_{k}\right)$ is bounded.

Motivated by the works of Chidume, Romanus and Nnyaba [17], Gebrie and Wangkeeree [19], Ogbuisi [26] and Shukla and Pant [31], we study SEEP (1.1)-(1.2) in the frame work of 2-uniformly convex and uniformly smooth Banach spaces. Our contributions in this paper are that:

(1) We consider a projected subgradient proximal method for split equality equilibrium problem in 2-uniformly convex Banach spaces which is uniformly smooth while the results of Gebrie and Wangkeeree [19] and Shukla and Pant [31] are restricted to Hilbert space.

(2) The monotonicity assumption imposed on the bifunctions in Chidume, Romanus and Nnyaba [17], Ogbuisi [26] and Shukla and Pant [31] is relaxed by assuming that the bifunctions in this paper are pseudomonotone. We also improve the results in Gebrie and Wangkeeree [19]. In Gebrie and Wangkeeree [19], they assumed that one of the bifunctions is pseudomomotone and the other bifunction is monotone. For example, take $f_{2}:(0, \infty) \times(0, \infty) \rightarrow \mathbb{R}, f_{2}(x, y):=\frac{1}{1+x}(y-$ $x), x, y \in(0, \infty)$. It is easy to see that $f_{2}$ is pseudomonotone but not monotone on $(0, \infty)$.

(3) The results of this paper generalize the results in Gebrie and Wangkeeree [19] and Ogbuisi [26] and other variant results on the split equilibrium problem in the literature to the split equality equilibrium problem in Banach spaces.

\section{PRELIMINARIES}

Let $B_{E}=\{x \in E:\|x\|=1\}$. A Banach space $E$ is said to be strictly convex if for any $x, y \in B_{E}$ and $x \neq y$ implies $\frac{\|x+y\|}{2}<1$. $E$ is also said to be uniformly convex if for each $\varepsilon \in(0,2]$, there exists $\delta>0$ such that for any $x, y \in B_{E},\|x-y\| \geq \varepsilon$ implies $\frac{\|x+y\|}{2} \leq 1-\delta$. The modulus of convexity of $E$ is the function $\delta_{E}:(0,2] \rightarrow[0,1]$ defined by

$$
\delta_{E}(\varepsilon):=\inf \left\{1-\left\|\frac{x+y}{2}\right\|: x, y \in B_{E} ; \varepsilon=\|x-y\|\right\} .
$$

$E$ is uniformly convex if and only if $\delta_{E}(\varepsilon)>0$ for all $\varepsilon \in(0,2]$ and p-uniformly convex if there exists a $C_{p}>0$ such that $\delta_{E}(\varepsilon) \geq C_{p} \varepsilon^{p}$ for any $\varepsilon \in(0,2]$. Clearly, every a p-uniformly convex Banach space is uniformly convex. For example, see [32] for more details.

A Banach space $E$ is said to be smooth if

$$
\lim _{t \rightarrow 0} \frac{\|x+t y\|-\|x\|}{t}
$$

exists for all $x, y \in B_{E}$. It is also said to be uniformly smooth if the limit is attained uniformly for $x, y \in$ $B_{E}$. It is well known that Hilbert and the Lebesgue $L_{p}(1<p \leq 2)$ spaces are 2-uniformly convex and uniformly smooth.

The normalised duality mapping $J_{E}: E \rightarrow 2^{E^{*}}$ is defined by

$$
J_{E}(x):=\left\{x^{*} \in E^{*}:\left\langle x, x^{*}\right\rangle=\|x\|^{2}=\left\|x^{*}\right\|^{2}\right\}, \forall x \in E .
$$

Let $E$ be a reflexive, strictly convex, smooth Banach space and let $J$ be the normalised duality mapping from $E$ into $E^{*}$. Then $J_{E}^{-1}$ is also single-valued, one-to-one, surjective, and is the duality mapping from $E^{*}$ into $E$. The normalised duality mapping $J_{E}$ possesses the following properties [3]:

(1) If $E$ is a smooth Banach space, then $J_{E}$ is single-valued.

(2) If $E$ is a strictly convex Banach space, then $J_{E}$ is one-to-one and strictly monotone. 
(3) If $E$ is a uniformly smooth Banach space, then $J_{E}$ is uniformly norm-to-norm continuous on each bounded subset of $E$.

(4) If $\mathrm{E}$ is a smooth, strictly convex and reflexive Banach space, then $J_{E}$ is single-valued, one-to-one and onto.

Let $E$ be a smooth Banach space. Alber [2] introduced the following Lyapunov functional

$$
\phi(x, y)=\|x\|^{2}-2\left\langle x, J_{E}(y)\right\rangle+\|y\|^{2} .
$$

It can be seen from the definition that $\phi$ satisfies the following conditions.

$$
(\|x\|-\|y\|)^{2} \leq \phi(x, y) \leq(\|x\|+\|y\|)^{2} .
$$

Lemma 2.1. [2, 4] Let E be a real uniformly convex and smooth Banach space. Then, the following identities hold:

1. $\phi(x, y)=\phi(x, z)+\phi(z, y)+2\left\langle x-z, J_{E}(z)-J_{E}(y)\right\rangle$.

2. $\phi(x, y)+\phi(y, x)=2\left\langle x-y, J_{E} x-J_{E} y\right\rangle ; \forall x, y \in E$.

If $E$ is a strictly convex and smooth Banach space, then for $x, y \in E, \phi(y, x)=0$ if and only if $x=y$ (see Remark 2.1 in [22]).

Lemma 2.2. [22] Let $E$ be a uniformly convex and smooth Banach space and let $\left\{x_{n}\right\}$ and $\left\{y_{n}\right\}$ be two sequences in $E$. If $\phi\left(x_{n}, y_{n}\right) \rightarrow 0$, and either $\left\{x_{n}\right\}$ or $\left\{y_{n}\right\}$ is bounded, then $\left\|x_{n}-y_{n}\right\| \rightarrow 0$.

Let $C$ be a nonempty, closed and convex subset of a reflexive, strictly convex and smooth Banach space $E$. Then for each $x \in E$ (see Alber [2]), there exists a unique element $x_{0} \in C$ (denoted by $\Pi_{C}(x)$ ) such that $\phi\left(x_{0}, x\right)=\min _{y \in C} \phi(y, x)$. The mapping $\Pi_{C}: E \rightarrow C$, defined by $\Pi_{C}(x)=x_{0}$, is called the generalized projection operator from $E$ onto $C$ and $x_{0}$ is called the generalized projection of $x$. In a Hilbert space, $\Pi_{C}=P_{C}$ (the metric projection operator).

Lemma 2.3. [22] Let $C$ be a nonempty closed and convex subset of a smooth Banach space $E$ and $x \in E$. Then, $x_{0}=\Pi_{C}(x)$ if and only if $\left\langle x_{0}-y, J_{E}(x)-J_{E}\left(x_{0}\right)\right\rangle \geq 0, \forall y \in C$.

Lemma 2.4. [22] Let $E$ be a reflexive, strictly convex and smooth Banach space, let $C$ be a nonempty closed and convex subset of $E$ and let $x \in E$. Then $\phi\left(y, \Pi_{C}(x)\right)+\phi\left(\Pi_{C}(x), x\right) \leq \phi(y, x), \forall y \in C$.

Lemma 2.5. [24] Let $E$ be a 2-uniformly convex and uniformly smooth Banach space. Then for every $x, y \in E, \phi(x, y) \geq \theta\|x-y\|^{2}$, where $\theta>0$ is the 2-uniformly convexity constant of $E$.

Lemma 2.6. [34] Let E be a real Banach space. Then the following are equivalent.

1. E is 2-uniformly smooth.

2. There exists a constant $D>0$ such that

$$
\|x+y\|^{2} \leq\|x\|^{2}+2\left\langle y, J_{E}(x)\right\rangle+2 D^{2}\|y\|^{2}, \quad \forall x, y \in E,
$$

where $D$ is the 2-uniformly smooth constant of $E$. In Hilbert spaces, $D=\frac{1}{\sqrt{2}}$.

Lemma 2.7. [7] Let $\frac{1}{p}+\frac{1}{q}=1, p, q>1$. A Banach space $E$ is $q$-uniformly smooth if and only if its dual $E^{*}$ is p-uniformly convex. 
Let $C$ be a nonempty closed and convex subset of a reflexive Banach space $E$. Let $f: C \times C \rightarrow \mathbb{R}$ be a bifunction where $f(x, \cdot)$ is a convex function for each $x \in C$. Then the $\varepsilon$-subdifferential ( $\varepsilon$-diagonal subdifferential) of $f$ at $x$, denoted by $\partial_{\varepsilon} f(x, \cdot)(x)$ is given by

$$
\partial_{\varepsilon} f(x, \cdot)(x)=\left\{w \in E^{*}: f(x, y)-f(x, x)+\varepsilon \geq\langle w, y-x\rangle, \forall y \in C\right\} .
$$

\section{Proposed Method}

Let $E_{1}, E_{2}$ and $E_{3}$ be 2-uniformly convex Banach spaces which are uniformly smooth and let $C_{1}$ and $C_{2}$ be nonempty closed and convex subsets of $E_{1}$ and $E_{2}$ respectively. We assume that $f_{1}$ and $f_{2}$ satisfy Condition $\mathrm{B}$ above and $D_{1}, D_{2}$ the 2-uniformly smooth constants of $E_{1}^{*}, E_{2}^{*}$ respectively. Throughout this paper, we also assume that $S \neq \emptyset$.

We now describe the iterative method we proposed for solving SEEP (1.1)-(1.2).

\section{Algorithm 1}

1: Initialization: For each $i=1,2$, pick $x_{0}^{(i)} \in C_{i}$ and choose $\left\{\rho_{k}^{(i)}\right\},\left\{\beta_{k}^{(i)}\right\},\left\{\delta_{k}^{(i)}\right\},\left\{\varepsilon_{k}^{(i)}\right\}$ and $\left\{\mu_{k}\right\}$ such that $\rho_{k}^{(i)}>\rho^{(i)}>0, \beta_{k}^{(i)} \geq 0, \varepsilon_{k}^{(i)} \geq 0,0<a<\delta_{k}^{(i)}<b<1,0<\lambda \leq \mu_{k} \leq \gamma<\frac{1}{\left(D_{1}^{2}\left\|A_{1}\right\|^{2}+D_{2}^{2}\left\|A_{2}\right\|^{2}\right)}$, $\sum_{k=0}^{\infty} \frac{\beta_{k}^{(i)}}{\rho_{k}^{(i)}}=\infty, \sum_{k=0}^{\infty} \frac{\beta_{k}^{(i)} \varepsilon_{k}^{(i)}}{\rho_{k}^{(i)}}<\infty$ and $\sum_{k=0}^{\infty}\left(\beta_{k}^{(i)}\right)^{2}<\infty$.

2: Find $w_{k}^{(i)} \in E_{i}^{*},(i=1,2)$ such that

$$
w_{k}^{i} \in \partial_{\varepsilon_{k}^{(i)}} f_{i}\left(x_{k}^{(i)}, \cdot\right)\left(x_{k}^{(i)}\right) .
$$

Let $\eta_{k}^{(i)}=\max \left\{\rho_{k}^{(i)},\left\|w_{k}^{(i)}\right\|\right\}$ and $\alpha_{k}^{(i)}=\frac{\beta_{k}^{(i)}}{\eta_{k}^{(i)}}$

3: Compute

$$
\left\{\begin{array}{l}
y_{k}^{(1)}=\Pi_{C_{1}} J_{E_{1}}^{-1}\left(J_{E_{1}} x_{k}^{(1)}-\alpha_{k}^{(1)} w_{k}^{(1)}\right) \\
y_{k}^{(2)}=\Pi_{C_{2}} J_{E_{1}}^{-1}\left(J_{E_{2}} x_{k}^{(2)}-\alpha_{k}^{(2)} w_{k}^{(2)}\right)
\end{array}\right.
$$

4: Compute

5: Compute

$$
\left\{\begin{array}{c}
t_{k}^{(1)}=J_{E_{1}}^{-1}\left(\delta_{k}^{(1)} J_{E_{1}} x_{k}^{(1)}+\left(1-\delta_{k}^{(1)}\right) J_{E_{1}} y_{k}^{(1)}\right), \\
t_{k}^{(2)}=J_{E_{2}}^{-1}\left(\delta_{k}^{(2)} J_{E_{2}} x_{k}^{(2)}+\left(1-\delta_{k}^{(2)}\right) J_{E_{2}} y_{k}^{(2)}\right) .
\end{array}\right.
$$

$$
\left\{\begin{array}{l}
x_{k+1}^{(1)}=\Pi_{C_{1}} J_{E_{1}}^{-1}\left(J_{E_{1}} t_{k}^{(1)}-\mu_{k} A_{1}^{*} J_{E_{3}}\left(A_{1} t_{k}^{(1)}-A_{2} t_{k}^{(2)}\right),\right. \\
x_{k+1}^{(2)}=\Pi_{C_{2}} J_{E_{2}}^{-1}\left(J_{E_{2}} t_{k}^{(2)}+\mu_{k} A_{2}^{*} J_{E_{3}}\left(A_{1} t_{k}^{(1)}-A_{2} t_{k}^{(2)}\right) .\right.
\end{array}\right.
$$

6: Set $\mathrm{k}:=\mathrm{k}+1$ and go to 2 .

\section{MAIN RESULTS}

Lemma 4.1. Let $\left\{y_{k}^{(1)}\right\},\left\{t_{k}^{(1)}\right\},\left\{x_{k}^{(1)}\right\},\left\{y_{k}^{(2)}\right\},\left\{t_{k}^{(2)}\right\}$ and $\left\{x_{k}^{(2)}\right\}$ be sequences generated by the Algorithm 1. For $\left(x^{*}, y^{*}\right) \in S$, we have

$$
\begin{aligned}
\phi\left(x^{*}, t_{k}^{(1)}\right) \leq & \phi\left(x^{*}, x_{k}^{(1)}\right)+2 \alpha_{k}^{(1)}\left(1-\delta_{k}^{(1)}\right) f_{1}\left(x_{k}^{(1)}, x^{*}\right) \\
& -\left(1-\delta_{k}^{(1)}\right) \phi\left(y_{k}^{(1)}, x_{k}^{(1)}\right)+\xi_{k}^{(1)}
\end{aligned}
$$


and

$$
\begin{aligned}
\phi\left(y^{*}, t_{k}^{(2)}\right) \leq & \phi\left(y^{*}, x_{k}^{(2)}\right)+2 \alpha_{k}^{(2)}\left(1-\delta_{k}^{(2)}\right) f_{2}\left(x_{k}^{(2)}, y^{*}\right) \\
& -\left(1-\delta_{k}^{(2)}\right) \phi\left(y_{k}^{(2)}, x_{k}^{(2)}\right)+\xi_{k}^{(2)}
\end{aligned}
$$

where

$$
\xi_{k}^{(i)}=2\left(1-\delta_{k}^{(i)}\right) \frac{\beta_{k}^{(i)} \varepsilon_{k}^{(i)}}{\rho_{k}^{(i)}}+2\left(1-\delta_{k}^{(i)}\right) \frac{\left(\beta_{k}^{(i)}\right)^{2}}{\theta_{i}}
$$

for $i=1,2$.

Proof. From $y_{k}^{(1)}=\Pi_{C_{1}} J_{E_{1}}^{-1}\left(J_{E_{1}} x_{k}^{(1)}-\alpha_{k}^{(1)} w_{k}^{(1)}\right)$, we have

$$
\left\langle J_{E_{1}} x_{k}^{(1)}-\alpha_{k}^{(1)} w_{k}^{(1)}-J_{E_{1}} y_{k}^{(1)}, y_{k}^{(1)}-x^{*}\right\rangle \geq 0 .
$$

Thus

$$
\begin{aligned}
\left\langle x^{*}-y_{k}^{(1)}, J_{E_{1}} x_{k}^{(1)}-J_{E_{1}} y_{k}^{(1)}\right\rangle & \leq \alpha_{k}^{(1)}\left\langle w_{k}^{(1)}, x^{*}-y_{k}^{(1)}\right\rangle \\
& =\alpha_{k}^{(1)}\left\langle w_{k}^{(1)}, x^{*}-x_{k}^{(1)}\right\rangle+\alpha_{k}^{(1)}\left\langle w_{k}^{(1)}, x_{k}^{(1)}-y_{k}^{(1)}\right\rangle \\
& \leq \alpha_{k}^{(1)}\left\langle w_{k}^{(1)}, x^{*}-x_{k}^{(1)}\right\rangle+\alpha_{k}^{(1)}\left\|w_{k}^{(1)}\right\|\left\|x_{k}^{(1)}-y_{k}^{(1)}\right\| .
\end{aligned}
$$

Moreover, since $x_{k}^{(1)} \in C_{1}$, we have

$$
\left\langle J_{E_{1}} x_{k}^{(1)}-\alpha_{k}^{(1)} w_{k}^{(1)}-J_{E_{1}} y_{k}^{(1)}, y_{k}^{(1)}-x_{k}^{(1)}\right\rangle \geq 0 .
$$

Therefore, it follows from Lemma 2.5, Lemma 2.1(2) and (4.4) that

$$
\begin{aligned}
2 \theta_{1}\left\|x_{k}^{(1)}-y_{k}^{(1)}\right\|^{2} & \leq \phi\left(x_{k}^{(1)}, y_{k}^{(1)}\right)+\phi\left(y_{k}^{(1)}, x_{k}^{(1)}\right) \\
& =2\left\langle J_{E_{1}} x_{k}^{(1)}-J_{E_{1}} y_{k}^{(1)}, x_{k}^{(1)}-y_{k}^{(1)}\right\rangle \\
& \leq 2 \alpha_{k}^{(1)}\left\langle w_{k}^{(1)}, x_{k}^{(1)}-y_{k}^{(1)}\right\rangle \\
& \leq 2 \alpha_{k}^{(1)}\left\|w_{k}^{(1)}\right\|\left\|x_{k}^{(1)}-y_{k}^{(1)}\right\| .
\end{aligned}
$$

From (4.5), we obtain

$$
\left\|x_{k}^{(1)}-y_{k}^{(1)}\right\| \leq \frac{\alpha_{k}^{(1)}}{\theta_{1}}\left\|w_{k}^{(1)}\right\|
$$

Thus,

$$
\begin{aligned}
\alpha_{k}^{(1)}\left\|w_{k}^{(1)}\right\|\left\|x_{k}^{(1)}-y_{k}^{(1)}\right\| & \leq \frac{1}{\theta_{1}}\left(\alpha_{k}^{(1)}\left\|w_{k}^{(1)}\right\|\right)^{2} \\
& =\frac{1}{\theta_{1}}\left(\frac{\beta_{k}^{(1)}}{\eta_{k}^{(1)}}\left\|w_{k}^{(1)}\right\|\right)^{2} \\
& =\frac{\left(\beta_{k}^{(1)}\right)^{2}}{\theta_{1}}\left(\frac{\left\|w_{k}^{(1)}\right\|}{\max \left\{\rho_{k}^{(1)},\left\|w_{k}^{(1)}\right\|\right\}}\right)^{2} \leq \frac{\left(\beta_{k}^{(1)}\right)^{2}}{\theta_{1}} .
\end{aligned}
$$

Since $x_{k}^{(1)} \in C_{1}$ and $w_{k}^{(1)} \in \partial_{\varepsilon_{k}^{(1)}} f_{1}\left(x_{k}^{(1)},.\right)\left(x_{k}^{(1)}\right)$, we have

$$
\begin{aligned}
f_{1}\left(x_{k}^{(1)}, x^{*}\right)+\varepsilon_{k}^{(1)} & =f_{1}\left(x_{k}^{(1)}, x^{*}\right)-f_{1}\left(x_{k}^{(1)}, x_{k}^{(1)}\right)+\varepsilon_{k}^{(1)} \\
& \geq\left\langle w_{k}^{(1)}, x^{*}-x_{k}^{(1)}\right\rangle .
\end{aligned}
$$


Using the definitions of $\alpha_{k}^{(1)}$ and $\eta_{k}^{(1)}$, we obtain

$$
\alpha_{k}^{(1)}=\frac{\beta_{k}^{(1)}}{\eta_{k}^{(1)}}=\frac{\beta_{k}^{(1)}}{\max \left\{\rho_{k}^{(1)},\left\|w_{k}^{(1)}\right\|\right\}} \leq \frac{\beta_{k}^{(1)}}{\rho_{k}^{(1)}} .
$$

From (4.3)-(4.9), we have

$$
\left\langle x^{*}-y_{k}^{(1)}, J_{E_{1}} x_{k}^{(1)}-J_{E_{1}} y_{k}^{(1)}\right\rangle \leq \alpha_{k}^{(1)} f_{1}\left(x_{k}^{(1)}, x^{*}\right)+\frac{\beta_{k}^{(1)} \varepsilon_{k}^{(1)}}{\rho_{k}^{(1)}}+\frac{\left(\beta_{k}^{(1)}\right)^{2}}{\theta_{1}} .
$$

By Lemma 2.1(1), we have

$$
2\left\langle x^{*}-y_{k}^{(1)}, J_{E_{1}} x_{k}^{(1)}-J_{E_{1}} y_{k}^{(1)}\right\rangle=\phi\left(x^{*}, y_{k}^{(1)}\right)+\phi\left(y_{k}^{(1)}, x_{k}^{(1)}\right)-\phi\left(x^{*}, x_{k}^{(1)}\right) .
$$

Combining (4.10) and (4.11), we have

$$
\begin{aligned}
\phi\left(x^{*}, y_{k}^{(1)}\right) \leq & \phi\left(x^{*}, x_{k}^{(1)}\right)-\phi\left(y_{k}^{(1)}, x_{k}^{(1)}\right) \\
& +2 \alpha_{k}^{(1)} f_{1}\left(x_{k}^{(1)}, x^{*}\right)+\frac{2 \beta_{k}^{(1)} \varepsilon_{k}^{(1)}}{\rho_{k}^{(1)}}+\frac{2\left(\beta_{k}^{(1)}\right)^{2}}{\theta_{1}} .
\end{aligned}
$$

Furthermore, by the definition of $t_{k}^{(1)}$, we have

$$
\begin{aligned}
\phi\left(x^{*}, t_{k}^{(1)}\right) & =\phi\left(x^{*}, J_{E_{1}}^{-1}\left(\delta_{k}^{(1)} J_{E_{1}} x_{k}^{(1)}+\left(1-\delta_{k}^{(1)}\right) J_{E_{1}} y_{k}^{(1)}\right)\right) \\
& \leq \delta_{k}^{(1)} \phi\left(x^{*}, x_{k}^{(1)}\right)+\left(1-\delta_{k}^{(1)}\right) \phi\left(x^{*}, y_{k}^{(1)}\right) .
\end{aligned}
$$

It then follows from (4.12) and (4.13) that

$$
\begin{aligned}
\phi\left(x^{*}, t_{k}^{(1)}\right) \leq & \delta_{k}^{(1)} \phi\left(x^{*}, x_{k}^{(1)}\right)+\left(1-\delta_{k}^{(1)}\right)\left[\phi\left(x^{*}, x_{k}^{(1)}\right)-\phi\left(y_{k}^{(1)}, x_{k}^{(1)}\right)\right. \\
& \left.+2 \alpha_{k}^{(1)} f_{1}\left(x_{k}^{(1)}, x^{*}\right)+\frac{2 \beta_{k}^{(1)} \varepsilon_{k}^{(1)}}{\rho_{k}^{(1)}}+\frac{2\left(\beta_{k}^{(1)}\right)^{2}}{\theta_{1}}\right]
\end{aligned}
$$

which means

$$
\phi\left(x^{*}, t_{k}^{(1)}\right) \leq \phi\left(x^{*}, x_{k}^{(1)}\right)+2 \alpha_{k}^{(1)} f_{1}\left(x_{k}^{(1)}, x^{*}\right)-\left(1-\delta_{k}^{(1)}\right) \phi\left(y_{k}^{(1)}, x_{k}^{(1)}\right)+\xi_{k}^{1} .
$$

Similarly, we have

$$
\phi\left(y^{*}, t_{k}^{(2)}\right) \leq \phi\left(y^{*}, x_{k}^{(2)}\right)+2 \alpha_{k}^{(2)} f_{2}\left(x_{k}^{(2)}, y^{*}\right)-\left(1-\delta_{k}^{(2)}\right) \phi\left(y_{k}^{(2)}, x_{k}^{(2)}\right)+\xi_{k}^{2} .
$$

Lemma 4.2. Let $\left\{y_{k}^{(1)}\right\},\left\{y_{k}^{(2)}\right\},\left\{t_{k}^{(1)}\right\},\left\{t_{k}^{(2)}\right\},\left\{x_{k}^{(1)}\right\}$ and $\left\{x_{k}^{(2)}\right\}$ be the sequences generated by the Algorithm 1. Let $\left(x^{*}, y^{*}\right) \in S$. Then

$$
\begin{aligned}
\phi\left(x^{*}, x_{k+1}^{(1)}\right)+\phi\left(y^{*}, x_{k+1}^{(2)}\right) \leq & \phi\left(x^{*}, x_{k}^{(1)}\right)+\phi\left(y^{*}, x_{k}^{(2)}\right)+2\left(1-\delta_{k}^{(1)}\right) \alpha_{k}^{(1)} f_{1}\left(x_{k}^{(1)}, x^{*}\right) \\
& +2\left(1-\delta_{k}^{(2)}\right) \alpha_{k}^{(2)} f_{2}\left(x_{k}^{(2)}, y^{*}\right)+\xi_{k}^{(1)}+\xi_{k}^{(1)}-K_{k},
\end{aligned}
$$

where

$K_{k}=\left(1-\delta_{k}^{(1)}\right) \phi\left(y_{k}^{(1)}, x_{k}^{(1)}\right)+\left(1-\delta_{k}^{(2)}\right) \phi\left(y_{k}^{(2)}, x_{k}^{(2)}\right)+2 \mu_{k}\left[1-\mu_{k}\left(D_{1}^{2}\left\|A_{1}\right\|^{2}+D_{2}^{2}\left\|A_{2}\right\|^{2}\right)\right]\left\|A_{1} t_{k}^{(1)}-A_{2} t_{k}^{(2)}\right\|^{2}$. 
Proof.

$$
\begin{aligned}
\phi\left(x^{*}, x_{k+1}^{(1)}\right)= & \phi\left(x^{*}, \Pi_{C_{1}} J_{E_{1}}^{-1}\left(J_{E_{1}} t_{k}^{(1)}-\mu_{k} A_{1}^{*} J_{E_{3}}\left(A_{1} t_{k}^{(1)}-A_{2} t_{k}^{(2)}\right)\right)\right) \\
\leq & \phi\left(x^{*}, J_{E_{1}}^{-1}\left(J_{E_{1}} t_{k}^{(1)}-\mu_{k} A_{1}^{*} J_{E_{3}}\left(A_{1} t_{k}^{(1)}-A_{2} t_{k}^{(2)}\right)\right)\right) \\
= & \left\|J_{E_{1}} t_{k}^{(1)}-\mu_{k} A_{1}^{*} J_{E_{3}}\left(A_{1} t_{k}^{(1)}-A_{2} t_{k}^{(2)}\right)\right\|^{2}-2\left\langle x^{*}, J_{E_{1}} t_{k}^{(1)}\right\rangle \\
& +2\left\langle x^{*}, \mu_{k} A_{1}^{*} J_{E_{3}}\left(A_{1} t_{k}^{(1)}-A_{2} t_{k}^{(2)}\right)\right\rangle+\left\|x^{*}\right\|^{2} \\
= & \left\|x^{*}\right\|^{2}-2\left\langle x^{*}, J_{E_{1}} t_{k}^{(1)}\right\rangle+\left\|t_{k}^{(1)}\right\|^{2}-2 \mu_{k}\left\langle A_{1} t_{k}^{(1)}, J_{E_{3}}\left(A_{1} t_{k}^{(1)}-A_{2} t_{k}^{(2)}\right)\right\rangle \\
& +2 \mu_{k}\left\langle A_{1} x^{*}, J_{E_{3}}\left(A_{1} t_{k}^{(1)}-A_{2} t_{k}^{(2)}\right)\right\rangle+2 \mu_{k}^{2} D_{1}^{2}\left\|A_{1}\right\|^{2}\left\|A_{1} t_{k}^{(1)}-A_{2} t_{k}^{(2)}\right\|^{2} \\
= & \phi\left(x^{*}, t_{k}^{(1)}\right)+2 \mu_{k}\left\langle A_{1} x^{*}-A_{1} t_{k}^{(1)}, J_{E_{3}}\left(A_{1} t_{k}^{(1)}-A_{2} t_{k}^{(2)}\right)\right\rangle \\
& +2 \mu_{k}^{2} D_{1}^{2}\left\|A_{1}\right\|^{2}\left\|A_{1} t_{k}^{(1)}-A_{2} t_{k}^{(2)}\right\|^{2} .
\end{aligned}
$$

Similarly, we have

$$
\begin{aligned}
\phi\left(y^{*}, x_{k+1}^{(2)}\right) \leq & \phi\left(y^{*}, t_{k}^{(2)}\right)+2 \mu_{k}\left\langle A_{2} t_{k}^{(2)}-A_{2} y^{*}, J_{E_{3}}\left(A_{1} t_{k}^{(1)}-A_{2} t_{k}^{(2)}\right)\right\rangle \\
& +2 \mu_{k}^{2} D_{2}^{2}\left\|A_{2}\right\|^{2}\left\|A_{1} t_{k}^{(1)}-A_{2} t_{k}^{(2)}\right\|^{2} .
\end{aligned}
$$

Adding (4.18) and (4.19) and noting that $A_{1} x^{*}=A_{2} y^{*}$, we obtain

$$
\begin{aligned}
\phi\left(x^{*}, x_{k+1}^{(1)}\right)+\phi\left(y^{*}, x_{k+1}^{(2)}\right) \leq & \phi\left(x^{*}, t_{k}^{(1)}\right)+\phi\left(y^{*}, t_{k}^{(2)}\right) \\
& +2 \mu_{k}\left\langle A_{2} t_{k}^{(2)}-A_{1} t_{k}^{(1)}, J_{E_{3}}\left(A_{1} t_{k}^{(1)}-A_{2} t_{k}^{(2)}\right)\right\rangle \\
& +2 \mu_{k}^{2}\left(D_{1}^{2}\left\|A_{1}\right\|^{2}+D_{2}^{2}\left\|A_{2}\right\|^{2}\right)\left\|A_{1} t_{k}^{(1)}-A_{2} t_{k}^{(2)}\right\|^{2} \\
= & \phi\left(x^{*}, t_{k}^{(1)}\right)+\phi\left(y^{*}, t_{k}^{(2)}\right)-2 \mu_{k}\left\|A_{1} t_{k}^{(1)}-A_{2} t_{k}^{(2)}\right\|^{2} \\
& +2 \mu_{k}^{2}\left(D_{1}^{2}\left\|A_{1}\right\|^{2}+D_{2}^{2}\left\|A_{2}\right\|^{2}\right)\left\|A_{1} t_{k}^{(1)}-A_{2} t_{k}^{(2)}\right\|^{2} \\
= & \phi\left(x^{*}, t_{k}^{(1)}\right)+\phi\left(y^{*}, t_{k}^{(2)}\right) \\
& -2 \mu_{k}\left[1-\mu_{k}\left(D_{1}^{2}\left\|A_{1}\right\|^{2}+D_{2}^{2}\left\|A_{2}\right\|^{2}\right)\right]\left\|A_{1} t_{k}^{(1)}-A_{2} t_{k}^{(2)}\right\|^{2} .
\end{aligned}
$$

From Lemma 4.1 and (4.20), we get

$$
\begin{aligned}
& \phi\left(x^{*}, x_{k+1}^{(1)}\right)+\phi\left(y^{*}, x_{k+1}^{(2)}\right) \\
\leq \quad & \phi\left(x^{*}, x_{k}^{(1)}\right)+\phi\left(y^{*}, x_{k}^{(2)}\right)+2 \alpha_{k}^{(1)}\left(1-\delta_{k}^{(1)}\right) f_{1}\left(x_{k}^{(1)}, x^{*}\right) \\
& -\left(1-\delta_{k}^{(1)}\right) \phi\left(y_{k}^{(1)}, x_{k}^{(1)}\right)+\xi_{k}^{(1)} \\
& +2 \alpha_{k}^{(2)}\left(1-\delta_{k}^{(2)}\right) f_{2}\left(x_{k}^{(2)}, y^{*}\right)-\left(1-\delta_{k}^{(2)}\right) \phi\left(y_{k}^{(2)}, x_{k}^{(2)}\right) \\
& +\xi_{k}^{(2)}-2 \mu_{k}\left[1-\mu_{k}\left(D_{1}^{2}\left\|A_{1}\right\|^{2}+D_{2}^{2}\left\|A_{2}\right\|^{2}\right)\right]\left\|A_{1} t_{k}^{(1)}-A_{2} t_{k}^{(2)}\right\|^{2} \\
= & \phi\left(x^{*}, x_{k}^{(1)}\right)+\phi\left(y^{*}, x_{k}^{(2)}\right)+2 \alpha_{k}^{(1)}\left(1-\delta_{k}^{(1)}\right) f_{1}\left(x_{k}^{(1)}, x^{*}\right) \\
& +2 \alpha_{k}^{(2)}\left(1-\delta_{k}^{(2)}\right) f_{2}\left(x_{k}^{(2)}, x^{*}\right)-K_{k}+\xi_{k}^{(1)}+\xi_{k}^{(2)} .
\end{aligned}
$$

Lemma 4.3. Let $\left\{y_{k}^{(1)}\right\},\left\{y_{k}^{(2)}\right\},\left\{x_{k}^{(1)}\right\},\left\{x_{k}^{(2)}\right\},\left\{t_{k}^{(1)}\right\}$ and $\left\{t_{k}^{(2)}\right\}$ be the sequences generated by the Algorithm 1. Then, for $\left(x^{*}, y^{*}\right) \in S$, 
i. The limit of the sequence $\left\{\phi\left(x^{*}, x_{k}^{(1)}\right)+\phi\left(y^{*}, x_{k}^{(2)}\right)\right\}$ exists and therefore $\left\{x_{k}^{(1)}\right\}$ and $\left\{x_{k}^{(2)}\right\}$ are bounded.

ii. $\limsup _{k \rightarrow \infty} f_{1}\left(x_{k}^{(1)}, x\right)=0$ and $\limsup _{k \rightarrow \infty} f_{2}\left(x_{k}^{(2)}, y\right)=0$ for all $(x, y) \in S$.

iii.

$$
\begin{gathered}
\lim _{k \rightarrow \infty}\left\|A_{1} t_{k}^{(1)}-A_{2} t_{k}^{(2)}\right\|=0, \\
\lim _{k \rightarrow \infty}\left\|y_{k}^{(1)}-x_{k}^{(1)}\right\|=\lim _{k \rightarrow \infty}\left\|y_{k}^{(2)}-x_{k}^{(2)}\right\|=0, \\
\lim _{k \rightarrow \infty}\left\|t_{k}^{(1)}-x_{k}^{(1)}\right\|=\lim _{k \rightarrow \infty}\left\|t_{k}^{(2)}-x_{k}^{(2)}\right\|=0 .
\end{gathered}
$$

Proof. $\quad$ i. Let $\left(x^{*}, y^{*}\right) \in S$. Since $f_{1}\left(x_{k}^{(1)}, x^{*}\right) \leq 0, f_{2}\left(x_{k}^{(2)}, y^{*}\right) \leq 0$, and $K_{k} \geq 0$, from Lemma 4.2, we have

$$
\phi\left(x^{*}, x_{k+1}^{(1)}\right)+\phi\left(y^{*}, x_{k+1}^{(2)}\right) \leq \phi\left(x^{*}, x_{k}^{(1)}\right)+\phi\left(y^{*}, x_{k}^{(2)}\right)+\xi_{k}^{(1)}+\xi_{k}^{(2)} .
$$

Observing that, for $i=1,2$,

$$
\begin{aligned}
\xi_{k}^{(i)} & =2\left(1-\delta_{k}^{(i)}\right) \frac{\beta_{k}^{(i)} \varepsilon_{k}^{(i)}}{\rho_{k}^{(i)}}+2\left(1-\delta_{k}^{(i)}\right) \frac{\left(\beta_{k}^{(i)}\right)^{2}}{\theta_{i}} \\
& \leq 2 \frac{\beta_{k}^{(i)} \varepsilon_{k}^{(i)}}{\rho_{k}^{(i)}}+2 \frac{\left(\beta_{k}^{(i)}\right)^{2}}{\theta_{i}}
\end{aligned}
$$

and using the initialization condition of the parameters, we can see that $\sum_{k=0}^{\infty} \xi_{k}^{(i)}<\infty, i=1,2$. Therefore, it follows (4.23) that $\lim _{k \rightarrow \infty}\left(\left(\phi\left(x^{*}, x_{k}^{(1)}\right)+\phi\left(y^{*}, x_{k}^{(2)}\right)\right)\right.$ exists and this implies that the sequences $\left\{x_{k}^{(1)}\right\}$ and $\left\{x_{k}^{(2)}\right\}$ are bounded.

ii. From Lemma 4.2, we have

$$
\begin{aligned}
& K_{k}+2\left(1-\delta_{k}^{(1)}\right) \alpha_{k}^{(1)}\left[-f_{1}\left(x_{k}^{(1)}, x^{*}\right)\right]+2\left(1-\delta_{k}^{(2)}\right) \alpha_{k}^{(2)}\left[-f_{2}\left(x_{k}^{(2)}, y^{*}\right)\right] \\
\leq & \left(\phi\left(x^{*}, x_{k}^{(1)}\right)+\phi\left(y^{*}, x_{k}^{(2)}\right)\right)-\left(\phi\left(x^{*}, x_{k+1}^{(1)}\right)+\phi\left(y^{*}, x_{k+1}^{(2)}\right)\right)+\xi_{k}^{(1)}+\xi_{k}^{(2)} \\
\leq & \left(\phi\left(x^{*}, x_{k}^{(1)}\right)+\phi\left(y^{*}, x_{k}^{(2)}\right)\right)-\left(\phi\left(x^{*}, x_{k+1}^{(1)}\right)+\phi\left(y^{*}, x_{k+1}^{(2)}\right)\right) \\
& +2 \frac{\beta_{k}^{(1)} \varepsilon_{k}^{(1)}}{\rho_{k}^{(1)}}+2 \frac{\left(\beta_{k}^{(1)}\right)^{2}}{\theta_{1}}+2 \frac{\beta_{k}^{(2)} \varepsilon_{k}^{(2)}}{\rho_{k}^{(2)}}+2 \frac{\left(\beta_{k}^{(2)}\right)^{2}}{\theta_{2}} .
\end{aligned}
$$

Summing up the above inequalities for every $N$, we obtain

$$
\begin{aligned}
0 \leq & \sum_{k=0}^{N}\left(K_{k}+2\left(1-\delta_{k}^{(1)}\right) \alpha_{k}^{(1)}\left[-f_{1}\left(x_{k}^{(1)}, x^{*}\right)\right]+2\left(1-\delta_{k}^{(2)}\right) \alpha_{k}^{(2)}\left[-f_{2}\left(x_{k}^{(2)}, y^{*}\right)\right]\right) \\
\leq & \sum_{k=0}^{N}\left[\left(\phi\left(x^{*}, x_{k}^{(1)}\right)+\phi\left(y^{*}, x_{k}^{(2)}\right)\right)-\left(\phi\left(x^{*}, x_{k+1}^{(1)}\right)+\phi\left(y^{*}, x_{k+1}^{(2)}\right)\right)\right. \\
& \left.+2 \frac{\beta_{k}^{(1)} \varepsilon_{k}^{(1)}}{\rho_{k}^{(1)}}+2 \frac{\left(\beta_{k}^{(1)}\right)^{2}}{\theta_{1}}+2 \frac{\beta_{k}^{(2)} \varepsilon_{k}^{(2)}}{\rho_{k}^{(2)}}+2 \frac{\left(\beta_{k}^{(2)}\right)^{2}}{\theta_{2}}\right],
\end{aligned}
$$


which gives

$$
\begin{aligned}
0 \leq & \sum_{k=0}^{N} K_{k}+\sum_{k=0}^{N} 2\left(1-\delta_{k}^{(1)}\right) \alpha_{k}^{(1)}\left[-f_{1}\left(x_{k}^{(1)}, x^{*}\right)\right] \\
& +\sum_{k=0}^{N} 2\left(1-\delta_{k}^{(2)}\right) \alpha_{k}^{(2)}\left[-f_{2}\left(x_{k}^{(2)}, y^{*}\right)\right] \\
\leq & \left(\phi\left(x^{*}, x_{0}^{(1)}\right)+\phi\left(y^{*}, x_{0}^{(2)}\right)\right)-\left(\phi\left(x^{*}, x_{N+1}^{(1)}\right)+\phi\left(y^{*}, x_{N+1}^{(2)}\right)\right) \\
& +2 \sum_{k=0}^{N} \frac{\beta_{k}^{(1)} \varepsilon_{k}^{(1)}}{\rho_{k}^{(1)}}+2 \sum_{k=0}^{N} \frac{\left(\beta_{k}^{(1)}\right)^{2}}{\theta_{1}}+2 \sum_{k=0}^{N} \frac{\beta_{k}^{(2)} \varepsilon_{k}^{(2)}}{\rho_{k}^{(2)}}+2 \sum_{k=0}^{N} \frac{\left(\beta_{k}^{(2)}\right)^{2}}{\theta_{2}} .
\end{aligned}
$$

Letting $N \rightarrow \infty$, we have

$$
\begin{aligned}
0 \leq & \sum_{k=0}^{\infty} K_{k}+\sum_{k=0}^{\infty} 2\left(1-\delta_{k}^{(1)}\right) \alpha_{k}^{(1)}\left[-f_{1}\left(x_{k}^{(1)}, x^{*}\right)\right] \\
& +\sum_{k=0}^{\infty} 2\left(1-\delta_{k}^{(2)}\right) \alpha_{k}^{(2)}\left[-f_{2}\left(x_{k}^{(2)}, y^{*}\right)\right]<\infty
\end{aligned}
$$

Hence,

$$
\begin{gathered}
\sum_{k=0}^{\infty} K_{k}<\infty, \\
\sum_{k=0}^{\infty} 2\left(1-\delta_{k}^{(1)}\right) \alpha_{k}^{(1)}\left[-f_{1}\left(x_{k}^{(1)}, x^{*}\right)\right]<\infty
\end{gathered}
$$

and

$$
\sum_{k=0}^{\infty} 2\left(1-\delta_{k}^{(2)}\right) \alpha_{k}^{(2)}\left[-f_{2}\left(x_{k}^{(2)}, y^{*}\right)\right]<\infty
$$

Since the sequence $\left\{x_{k}^{(1)}\right\}$ is bounded, by the Condition B (B6), the sequence $\left\{w_{k}^{(1)}\right\}$ is also bounded. Thus there exists a real number $w^{(1)} \geq \rho^{(1)}$ such that $\left\|w_{k}^{(1)}\right\| \leq w^{(1)}$. Therefore,

$$
\alpha_{k}^{(1)}=\frac{\beta_{k}^{(1)}}{\eta_{k}^{(1)}}=\frac{\beta_{k}^{(1)}}{\max \left\{\rho_{k}^{(1)},\left\|w_{k}^{(1)}\right\|\right\}}=\frac{\beta_{k}^{(1)}}{\rho_{k}^{(1)} \max \left\{1, \frac{\left\|w_{k}^{(1)}\right\|}{\rho_{k}^{(1)}}\right\}} \geq \frac{\beta_{k}^{(1)} \rho^{(1)}}{\rho_{k}^{(1)} w^{(1)}} .
$$

Note that

$$
\begin{aligned}
0 & <2(1-b) \sum_{k=0}^{\infty} \alpha_{k}^{(1)}\left[-f_{1}\left(x_{k}^{(1)}, x^{*}\right)\right] \\
& \leq \sum_{k=0}^{\infty} 2\left(1-\delta_{k}^{(2)}\right) \alpha_{k}^{(2)}\left[-f_{2}\left(x_{k}^{(2)}, y^{*}\right)\right]<\infty .
\end{aligned}
$$

From (4.31) and (4.32), we have

$$
\begin{aligned}
0 & <2(1-b) \sum_{k=0}^{\infty} \frac{\beta_{k}^{(1)} \rho^{(1)}}{\rho_{k}^{(1)} w^{(1)}}\left[-f_{1}\left(x_{k}^{(1)}, x^{*}\right)\right] \\
& \leq 2(1-b) \sum_{k=0}^{\infty} \alpha_{k}^{(1)}\left[-f_{1}\left(x_{k}^{(1)}, x^{*}\right)\right]<\infty .
\end{aligned}
$$


That is

$$
0 \leq \frac{2 \rho^{(1)}(1-b)}{w^{(1)}} \sum_{k=0}^{\infty} \frac{\beta_{k}^{(1)}}{\rho_{k}^{(1)}}\left[-f_{1}\left(x_{k}^{(1)}, x^{*}\right)\right]<\infty
$$

Similarly, we have

$$
0 \leq \frac{2 \rho^{(2)}(1-b)}{w^{(2)}} \sum_{k=0}^{\infty} \frac{\beta_{k}^{(2)}}{\rho_{k}^{(2)}}\left[-f_{2}\left(x_{k}^{(2)}, x^{*}\right)\right]<\infty
$$

Since $\sum_{k=0}^{\infty} \frac{\beta_{k}^{(i)}}{\rho_{k}^{(i)}}=\infty, i=1,2,-f_{1}\left(x^{*}, x_{k}^{(1)}\right) \leq 0$ and $-f_{2}\left(y^{*}, x_{k}^{(2)}\right) \leq 0$, we conclude that

$$
\limsup _{k \rightarrow \infty} f_{1}\left(x_{k}^{(1)}, x\right)=0 \text { and } \limsup _{k \rightarrow \infty} f_{2}\left(x_{k}^{(2)}, y\right)=0, \forall(x, y) \in S .
$$

iii. From (4.28), the conditions

$$
\mu_{k} \in(\lambda, \gamma) \subset\left(0, \frac{1}{D_{1}\left\|A_{1}\right\|^{2}+D_{2}\left\|A_{2}\right\|^{2}}\right)
$$

and $0<a<\delta_{k}^{i}<b<1, i=1,2$, we have

$$
\lim _{k \rightarrow \infty}\left\|A_{1} t_{k}^{(1)}-A_{2} t_{k}^{(2)}\right\|=0
$$

Also $\lim _{k \rightarrow \infty} \phi\left(y_{k}^{(1)}, x_{k}^{(1)}\right)=0$, which implies $\lim _{k \rightarrow \infty}\left\|x_{k}^{(1)}-y_{k}^{(1)}\right\|=0$.

Similarly, $\lim _{k \rightarrow \infty} \phi\left(y_{k}^{(2)}, x_{k}^{(2)}\right)=0$, and consequently $\lim _{k \rightarrow \infty}\left\|x_{k}^{(2)}-y_{k}^{(2)}\right\|=0$. Since $E_{1}$ is uniformly smooth, we have that the duality mapping $J_{E_{1}}$ is uniformly norm to norm continuous. From $\lim _{k \rightarrow \infty}\left\|x_{k}^{(1)}-y_{k}^{(1)}\right\|=0$, we have

$$
\begin{aligned}
\left\|J_{E_{(1)}} t_{k}^{(1)}-J_{E_{1}} x_{k}^{(1)}\right\| & =\left\|\delta_{k}^{(1)} J_{E_{1}} x_{k}^{(1)}+\left(1-\delta_{k}^{(1)}\right) J_{E_{1}} y_{k}^{(1)}-J_{E_{1}} x_{k}^{(1)}\right\| \\
& =\left(1-\delta_{k}^{(1)}\right)\left\|J_{E_{1}} y_{k}^{(1)}-J_{E_{1}} x_{k}^{(1)}\right\| \rightarrow 0, k \rightarrow \infty .
\end{aligned}
$$

Moreover, since $E_{1}$ is 2-uniformly convex, we have that $E_{1}^{*}$ is 2-uniformly smooth which implies it is uniformly smooth and thus $J_{E_{1}}^{-1}$ is uniformly norm to norm continuous.

Therefore,

$$
\left\|t_{k}^{(1)}-x_{k}^{(1)}\right\|=\left\|J_{E_{1}}^{-1} J_{E_{1}} t_{k}^{(1)}-J_{E_{1}}^{-1} J_{E_{1}} x_{k}^{(1)}\right\| \rightarrow 0, k \rightarrow \infty
$$

By the same line of argument, we have $\left\|t_{k}^{(2)}-x_{k}^{(2)}\right\| \rightarrow 0$ as $k \rightarrow \infty$.

Theorem 4.1. Assume that $f_{1}$ and $f_{2}$ satisfy condition $B$ and let $\left\{y_{k}^{(1)}\right\},\left\{y_{k}^{(2)}\right\},\left\{t_{k}^{(1)}\right\},\left\{t_{k}^{(2)}\right\}\left\{x_{k}^{(1)}\right\}$ and $x_{k}^{(2)}$ be the sequences generated by the Algorithm 1. Then the sequences $\left\{\left(y_{k}^{(1)}, y_{k}^{(2)}\right)\right\},\left\{\left(t_{k}^{(1)}, t_{k}^{(2)}\right)\right\}$ and $\left\{\left(x_{k}^{(1)}, x_{k}^{(2)}\right)\right\}$ converge strongly to $(p, q) \in S$.

Proof. Let $\left(x^{*}, y^{*}\right) \in S$. From Lemma 4.3(i), we see that the sequence $\left\{x_{k}^{(1)}\right\}$ and $\left\{x_{k}^{(2)}\right\}$ are bounded. Therefore, there exists a subsequence $\left\{x_{k_{j}}^{(1)}\right\}$ of $\left\{x_{k}^{(1)}\right\}$ such that $x_{k_{j}}^{(1)} \rightarrow p$, where $p \in C_{1}$ and

$$
\limsup _{j \rightarrow \infty} f_{1}\left(x_{k_{j}}^{(1)}, x^{*}\right)=\lim _{i \rightarrow \infty} f_{1}\left(x_{k_{i}}^{(1)}, x^{*}\right) .
$$


Also, there exists a subsequence $\left\{x_{k_{j}}^{(2)}\right\}$ of $\left\{x_{k}^{(2)}\right\}$ such that $x_{k_{j}}^{(2)} \rightarrow q$, where $q \in C_{2}$ and

$$
\limsup _{j \rightarrow \infty} f_{2}\left(x_{k_{j}}^{(2)}, x^{*}\right)=\lim _{i \rightarrow \infty} f_{2}\left(x_{k_{i}}^{(2)}, x^{*}\right) .
$$

By the weakly upper semicontinuity of $f_{1}\left(\cdot, x^{*}\right)$ and Lemma 4.3(ii), we have

$$
f_{1}\left(p, x^{*}\right) \geq \limsup _{j \rightarrow \infty} f_{1}\left(x_{k_{j}}^{(1)}, x^{*}\right)=\lim _{i \rightarrow \infty} f_{1}\left(x_{k_{i}}^{(1)}, x^{*}\right)=\limsup _{k \rightarrow \infty} f_{1}\left(x_{k}^{(1)}, x^{*}\right)=0 .
$$

Since $x^{*} \in E P\left(f_{1}, C_{1}\right)$ and $p \in C_{1}$, we have $f_{1}\left(x^{*}, p\right) \geq 0$. From the pseudomonotonicity of $f_{1}$, we have $f\left(p, x^{*}\right) \leq 0$. This together with (4.35) gives $f_{1}\left(x^{*}, p\right)=0$. Hence, by Condition B3, we have $p \in E P\left(f_{1}, C_{1}\right)$. Similarly, we obtain $q \in E P\left(f_{2}, C_{2}\right)$. By the fact that $\lim _{k \rightarrow \infty}\left\|x_{k}^{(i)}-t_{k}^{(i)}\right\|=0$, we have that $t_{k_{j}}^{(1)} \rightarrow p$ and $t_{k_{j}}^{(2)} \rightarrow q$. Moreover, since $A_{1}$ and $A_{2}$ are bounded linear operators, we have $A_{1} t_{k_{j}}^{(1)} \rightarrow A_{1} p$. and $A_{1} t_{k_{j}}^{(2)} \rightarrow A_{2} q$. Also, by weakly semi-continuity of norms, it follows that

$$
\left\|A_{1} p-A_{2} q\right\| \leq \liminf _{k \rightarrow \infty}\left\|A_{1} t_{k}^{(1)}-A_{2} t_{k}^{(2)}\right\|=0 .
$$

Hence, we have that $(p, q) \in S$ and $(p, q)$ is a weak cluster point of the sequence $\left\{\left(x_{k}^{(1)}, x_{k}^{(2)}\right)\right\}$. By Lemma 4.3, $\left\{\phi\left(p, x_{k}^{(1)}\right)+\phi\left(q, x_{k}^{(2)}\right)\right\}$ converges. Hence, we conclude that $\left\{\left(x_{k}^{(1)}, x_{k}^{(2)}\right)\right\}$ strongly converges to $(p, q)$.

We now give a convergence result which does not require the prior knowledge of the operator norm.

Lemma 4.4. Let $\left\{y_{k}^{(1)}\right\},\left\{y_{k}^{(2)}\right\},\left\{t_{k}^{(1)}\right\},\left\{t_{k}^{(2)}\right\},\left\{x_{k}^{(1)}\right\}$ and $\left\{x_{k}^{(2)}\right\}$ be the sequences generated by the Algorithm 1 but with the step size $\mu_{k}$ chosen as follows:

$$
\mu_{k} \in\left(\varepsilon, \frac{2\left\|A_{1} t_{k}^{(1)}-A_{2} t_{k}^{(2)}\right\|^{2}}{D_{1}^{2}\left\|A_{1}^{*} J_{E_{3}}\left(A_{1} t_{k}^{(1)}-A_{2} t_{k}^{(2)}\right)\right\|^{2}+D_{2}^{2}\left\|A_{2}^{*} J_{E_{3}}\left(A_{1} t_{k}^{(1)}-A_{2} t_{k}^{(2)}\right)\right\|^{2}}-\varepsilon\right), k \in \Omega,
$$

otherwise $\mu_{k}=\mu$ ( $\mu$ being any positive real number $)$, where $\Omega=\left\{k: A_{1} t_{k}^{(1)}-A_{2} t_{k}^{(2)} \neq 0\right\}$. Let $\left(x^{*}, y^{*}\right) \in S$. Then

$$
\begin{aligned}
\phi\left(x^{*}, x_{k+1}^{(1)}\right)+\phi\left(y^{*}, x_{k+1}^{(2)}\right) \leq & \phi\left(x^{*}, x_{k+1}^{(1)}\right)+\phi\left(y^{*}, x_{k+1}^{(2)}+2\left(1-\delta_{k}^{(1)}\right) \alpha_{k}^{(1)} f_{1}\left(x_{k}^{(1)}, x^{*}\right)\right. \\
& +2\left(1-\delta_{k}^{(2)}\right) \alpha_{k}^{(2)} f_{2}\left(x_{k}^{(2)}, x^{*}\right)+\xi_{k}^{(1)}+\xi_{k}^{(1)}-P_{k},
\end{aligned}
$$

where

$$
\begin{aligned}
P_{k}= & \left(1-\delta_{k}^{(1)}\right) \phi\left(y_{k}^{(1)}, x_{k}^{(1)}\right)+\left(1-\delta_{k}^{(2)}\right) \phi\left(y_{k}^{(2)}, x_{k}^{(2)}\right) \\
& +\varepsilon\left(D_{1}^{2}\left\|A_{1}^{*} J_{E_{3}}\left(A_{1} t_{k}^{(1)}-A_{2} t_{k}^{(2)}\right)\right\|^{2}+D_{2}^{2}\left\|A_{2}^{*} J_{E_{3}}\left(A_{1} t_{k}^{(1)}-A_{2} t_{k}^{(2)}\right)\right\|^{2}\right) .
\end{aligned}
$$

Proof. First we show that $\mu_{k}$ is well defined. Since $\left(x^{*}, y^{*}\right) \in S$, we have $A_{1} x^{*}=B y^{*}$.

Now

$$
\left\langle A_{1}^{*} J_{E_{3}}\left(A_{1} t_{k}^{(1)}-A_{2} t_{k}^{(2)}\right), t_{k}^{(1)}-x^{*}\right\rangle=\left\langle J_{E_{3}}\left(A_{1} t_{k}^{(1)}-A_{2} t_{k}^{(2)}\right), A_{1} t_{k}^{(1)}-A_{1} x^{*}\right\rangle
$$

and

$$
\left\langle A_{2}^{*} J_{E_{3}}\left(A_{1} t_{k}^{(1)}-A_{2} t_{k}^{(2)}\right), y^{*}-t_{k}^{(2)}\right\rangle=\left\langle J_{E_{3}}\left(A_{1} t_{k}^{(1)}-A_{2} t_{k}^{(2)}\right), A_{1} y^{*}-A_{1} t_{k}^{(2)}\right\rangle .
$$


Thus, adding (4.39) and (4.40), we obtain, $\forall k \in \Omega$,

$$
\begin{aligned}
\left\|A_{1} t_{k}^{(1)}-A_{2} t_{k}^{(2)}\right\|^{2}= & \left\langle A_{1}^{*} J_{E_{3}}\left(A_{1} t_{k}^{(1)}-A_{2} t_{k}^{(1)}\right), t_{k}^{(1)}-x^{*}\right\rangle \\
& +\left\langle A_{2}^{*} J_{E_{3}}\left(A_{1} t_{k}^{(1)}-A_{2} t_{k}^{(1)}\right), y^{*}-t_{k}^{(2)}\right\rangle \\
\leq & \left\|A_{1}^{*} J_{E_{3}}\left(A_{1} t_{k}^{(1)}-A_{2} t_{k}^{(1)}\right)\right\|\left\|t_{k}^{(1)}-x^{*}\right\| \\
& +\left\|A_{2}^{*} J_{E_{3}}\left(A_{1} t_{k}^{(1)}-A_{2} t_{k}^{(1)}\right)\right\|\left\|y^{*}-t_{k}^{(2)}\right\| .
\end{aligned}
$$

Therefore, for $k \in \Omega,\left\|A_{1} t_{k}^{(1)}-A_{2} t_{k}^{(2)}\right\|>0$. We have

$$
\left\|A_{1}^{*} J_{E_{3}}\left(A_{1} t_{k}^{(1)}-A_{2} t_{k}^{(1)}\right)\right\| \neq 0
$$

or

$$
\left\|A_{2}^{*} J_{E_{3}}\left(A_{1} t_{k}^{(1)}-A_{2} t_{k}^{(1)}\right)\right\| \neq 0 .
$$

Hence $\mu_{k}$ is well defined. Now,

$$
\begin{aligned}
\phi\left(x^{*}, x_{k+1}^{(1)}\right)= & \phi\left(x^{*}, \Pi_{C_{1}} J_{E_{1}}^{-1}\left(J_{E_{1}} t_{k}^{(1)}-\mu_{k} A_{1}^{*} J_{E_{3}}\left(A_{1} t_{k}^{(1)}-A_{2} t_{k}^{(2)}\right)\right)\right) \\
\leq & \phi\left(x^{*}, J_{E_{1}}^{-1}\left(J_{E_{1}} t_{k}^{(1)}-\mu_{k} A_{1}^{*} J_{E_{3}}\left(A_{1} t_{k}^{(1)}-A_{2} t_{k}^{(2)}\right)\right)\right) \\
= & \left\|J_{E_{1}} t_{k}^{(1)}-\mu_{k} A_{1}^{*} J_{E_{3}}\left(A_{1} t_{k}^{(1)}-A_{2} t_{k}^{(2)}\right)\right\|^{2}-2\left\langle x^{*}, J_{E_{1}} t_{k}^{(1)}\right\rangle \\
& +2\left\langle x^{*}, \mu_{k} A_{1}^{*} J_{E_{3}}\left(A_{1} t_{k}^{(1)}-A_{2} t_{k}^{(2)}\right)\right\rangle+\left\|x^{*}\right\|^{2} \\
= & \left\|x^{*}\right\|^{2}-2\left\langle x^{*}, J_{E_{1}} t_{k}^{(1)}\right\rangle+\left\|t_{k}^{(1)}\right\|^{2}-2 \mu_{k}\left\langle A_{1} t_{k}^{(1)}, J_{E_{3}}\left(A_{1} t_{k}^{(1)}-A_{2} t_{k}^{(2)}\right)\right\rangle \\
& +2 \mu_{k}\left\langle A_{1} x^{*}, J_{E_{3}}\left(A_{1} t_{k}^{(1)}-A_{2} t_{k}^{(2)}\right)\right\rangle+2 \mu_{k}^{2} D_{1}^{2}\left\|A_{1}^{*} J_{E_{3}}\left(A_{1} t_{k}^{(1)}-A_{2} t_{k}^{(2)}\right)\right\|^{2} \\
= & \phi\left(x^{*}, t_{k}^{(1)}\right)+2 \mu_{k}\left\langle A_{1} x^{*}-A_{1} t_{k}^{(1)}, J_{E_{3}}\left(A_{1} t_{k}^{(1)}-A_{2} t_{k}^{(2)}\right)\right\rangle \\
& +2 \mu_{k}^{2} D_{1}^{2}\left\|A_{1}^{*} J_{E_{3}}\left(A_{1} t_{k}^{(1)}-A_{2} t_{k}^{(2)}\right)\right\|^{2} .
\end{aligned}
$$

Similarly, we have

$$
\begin{aligned}
\phi\left(y^{*}, x_{k+1}^{(2)}\right) \leq & \phi\left(y^{*}, t_{k}^{(2)}\right)+2 \mu_{k}\left\langle A_{2} t_{k}^{(2)}-A_{2} y^{*}, J_{E_{3}}\left(A_{1} t_{k}^{(1)}-A_{2} t_{k}^{(2)}\right)\right\rangle \\
& +2 \mu_{k}^{2} D_{2}^{2}\left\|A_{2}^{*} J_{E_{3}}\left(A_{1} t_{k}^{(1)}-A_{2} t_{k}^{(2)}\right)\right\|^{2} .
\end{aligned}
$$

Adding (4.42) and (4.43) and noting that $A_{1} x^{*}=A_{2} y^{*}$, we obtain

$$
\begin{aligned}
\phi\left(x^{*}, x_{k+1}^{(1)}\right)+\phi\left(y^{*}, x_{k+1}^{(2)}\right) \leq & \phi\left(x^{*}, t_{k}^{(1)}\right)+\phi\left(y^{*}, t_{k}^{(2)}\right)+2 \mu_{k}\left\langle A_{2} t_{k}^{(2)}-A_{1} t_{k}^{(1)}, J_{E_{3}}\left(A_{1} t_{k}^{(1)}-A_{2} t_{k}^{(2)}\right)\right\rangle \\
& +2 \mu_{k}^{2} D_{1}^{2}\left\|A_{1}^{*} J_{E_{3}}\left(A_{1} t_{k}^{(1)}-A_{2} t_{k}^{(2)}\right)\right\|^{2}+2 \mu_{k}^{2} D_{2}^{2}\left\|A_{2}^{*} J_{E_{3}}\left(A_{1} t_{k}^{(1)}-A_{2} t_{k}^{(2)}\right)\right\|^{2} \\
= & \phi\left(x^{*}, t_{k}^{(1)}\right)+\phi\left(y^{*}, t_{k}^{(2)}\right)-2 \mu_{k}\left[\left\|A_{1} t_{k}^{(1)}-A_{2} t_{k}^{(2)}\right\|^{2}\right. \\
& +\mu_{k}\left(D_{1}^{2}\left\|A_{1}^{*} J_{E_{3}}\left(A_{1} t_{k}^{(1)}-A_{2} t_{k}^{(2)}\right)\right\|^{2}\right. \\
& \left.\left.+D_{2}^{2}\left\|A_{2}^{*} J_{E_{3}}\left(A_{1} t_{k}^{(1)}-A_{2} t_{k}^{(2)}\right)\right\|^{2}\right)\right] \\
= & \phi\left(x^{*}, t_{k}^{(1)}\right)+\phi\left(y^{*}, t_{k}^{(2)}\right)-2 \varepsilon\left(D_{1}^{2}\left\|A_{1}^{*} J_{E_{3}}\left(A_{1} t_{k}^{(1)}-A_{2} t_{k}^{(2)}\right)\right\|^{2}\right. \\
& \left.+D_{2}^{2}\left\|A_{2}^{*} J_{E_{3}}\left(A_{1} t_{k}^{(1)}-A_{2} t_{k}^{(2)}\right)\right\|^{2}\right) .
\end{aligned}
$$


From Lemma 4.1 and (4.44), we get

$$
\begin{aligned}
& \phi\left(x^{*}, x_{k+1}^{(1)}\right)+\phi\left(y^{*}, x_{k+1}^{(2)}\right) \\
\leq \quad & \phi\left(x^{*}, x_{k}^{(1)}\right)+\phi\left(y^{*}, x_{k}^{(2)}\right)+2 \alpha_{k}^{(1)}\left(1-\delta_{k}^{(1)}\right) f_{1}\left(x_{k}^{(1)}, x^{*}\right) \\
& -\left(1-\delta_{k}^{(1)}\right) \phi\left(y_{k}^{(1)}, x_{k}^{(1)}\right)+\xi_{k}^{(1)} \\
& +2 \alpha_{k}^{(2)}\left(1-\delta_{k}^{(2)}\right) f_{2}\left(x_{k}^{(2)}, y^{*}\right)-\left(1-\delta_{k}^{(2)}\right) \phi\left(y_{k}^{(2)}, x_{k}^{(2)}\right) \\
& +\xi_{k}^{(2)}-2 \varepsilon\left(D_{1}^{2}\left\|A_{1}^{*} J_{E_{3}}\left(A_{1} t_{k}^{(1)}-A_{2} t_{k}^{(2)}\right)\right\|^{2}+D_{2}^{2}\left\|A_{2}^{*} J_{E_{3}}\left(A_{1} t_{k}^{(1)}-A_{2} t_{k}^{(2)}\right)\right\|^{2}\right) \\
= & \phi\left(x^{*}, x_{k}^{(1)}\right)+\phi\left(y^{*}, x_{k}^{(2)}\right)+2 \alpha_{k}^{(1)}\left(1-\delta_{k}^{(1)}\right) f_{1}\left(x_{k}^{(1)}, x^{*}\right) \\
& +2 \alpha_{k}^{(2)}\left(1-\delta_{k}^{(2)}\right) f_{2}\left(x_{k}^{(2)}, y^{*}\right)-P_{k}+\xi_{k}^{(1)}+\xi_{k}^{(2)} .
\end{aligned}
$$

Lemma 4.5. Let $\left\{y_{k}^{(1)}\right\},\left\{y_{k}^{(2)}\right\},\left\{x_{k}^{(1)}\right\},\left\{x_{k}^{(2)}\right\},\left\{t_{k}^{(1)}\right\}$ and $\left\{t_{k}^{(2)}\right\}$ be the sequences generated by the Algorithm 1 and $\mu_{k}$ be as in Lemma 4.4. Then, for $\left(x^{*}, y^{*}\right) \in S$ :

$i$. The limit of the sequence $\left\{\phi\left(x^{*}, x_{k}^{(1)}\right)+\phi\left(y^{*}, x_{k}^{(2)}\right)\right\}$ exists and therefore $\left\{x_{k}^{(1)}\right\}$ and $\left\{x_{k}^{(2)}\right\}$ are bounded.

ii. $\limsup _{k \rightarrow \infty} f_{1}\left(x_{k}^{(1)}, x\right)=0$ and $\limsup _{k \rightarrow \infty} f_{2}\left(x_{k}^{(2)}, y\right)=0$ for all $(x, y) \in S$.

iii.

$$
\begin{gathered}
\lim _{k \rightarrow \infty}\left\|A_{1} t_{k}^{(1)}-A_{2} t_{k}^{(2)}\right\|=0, \\
\lim _{k \rightarrow \infty}\left\|y_{k}^{(1)}-x_{k}^{(1)}\right\|=\lim _{k \rightarrow \infty}\left\|y_{k}^{(2)}-x_{k}^{(2)}\right\|=0, \\
\lim _{k \rightarrow \infty}\left\|t_{k}^{(1)}-x_{k}^{(1)}\right\|=\lim _{k \rightarrow \infty}\left\|t_{k}^{(2)}-x_{k}^{(2)}\right\|=0 .
\end{gathered}
$$

Proof. $\quad$ i. The proof is similar to the proof of Lemma 4.3 with $K_{k}$ replaced with $P_{k}$ and Lemma 4.2 replaced by Lemma 4.4. Thus we omit the proof.

ii. From Lemma 4.4, we have

$$
\begin{aligned}
& P_{k}+2\left(1-\delta_{k}^{(1)}\right) \alpha_{k}^{(1)}\left[-f_{1}\left(x_{k}^{(1)}, x^{*}\right)\right]+2\left(1-\delta_{k}^{(2)}\right) \alpha_{k}^{(2)}\left[-f_{2}\left(x_{k}^{(2)}, y^{*}\right)\right] \\
\leq & \left(\phi\left(x^{*}, x_{k}^{(1)}\right)+\phi\left(y^{*}, x_{k}^{(2)}\right)\right)-\left(\phi\left(x^{*}, x_{k+1}^{(1)}\right)+\phi\left(y^{*}, x_{k+1}^{(2)}\right)\right)+\xi_{k}^{(1)}+\xi_{k}^{(2)} \\
\leq & \left(\phi\left(x^{*}, x_{k}^{(1)}\right)+\phi\left(y^{*}, x_{k}^{(2)}\right)\right)-\left(\phi\left(x^{*}, x_{k+1}^{(1)}\right)+\phi\left(y^{*}, x_{k+1}^{(2)}\right)\right) \\
& +2 \frac{\beta_{k}^{(1)} \varepsilon_{k}^{(1)}}{\rho_{k}^{(1)}}+2 \frac{\left(\beta_{k}^{(1)}\right)^{2}}{\theta_{1}}+2 \frac{\beta_{k}^{(2)} \varepsilon_{k}^{(2)}}{\rho_{k}^{(2)}}+2 \frac{\left(\beta_{k}^{(2)}\right)^{2}}{\theta_{2}} .
\end{aligned}
$$

Summing up the above inequalities for every $N$, we obtain

$$
\begin{aligned}
0 \leq & \sum_{k=0}^{N}\left(P_{k}+2\left(1-\delta_{k}^{(1)}\right) \alpha_{k}^{(1)}\left[-f_{1}\left(x_{k}^{(1)}, x^{*}\right)\right]+2\left(1-\delta_{k}^{(2)}\right) \alpha_{k}^{(2)}\left[-f_{2}\left(x_{k}^{(2)}, y^{*}\right)\right]\right) \\
\leq & \sum_{k=0}^{N}\left[\left(\phi\left(x^{*}, x_{k}^{(1)}\right)+\phi\left(y^{*}, x_{k}^{(2)}\right)\right)-\left(\phi\left(x^{*}, x_{k+1}^{(1)}\right)+\phi\left(y^{*}, x_{k+1}^{(2)}\right)\right)\right. \\
& \left.+2 \frac{\beta_{k}^{(1)} \varepsilon_{k}^{(1)}}{\rho_{k}^{(1)}}+2 \frac{\left(\beta_{k}^{(1)}\right)^{2}}{\theta_{1}}+2 \frac{\beta_{k}^{(2)} \varepsilon_{k}^{(2)}}{\rho_{k}^{(2)}}+2 \frac{\left(\beta_{k}^{(2)}\right)^{2}}{\theta_{2}}\right]
\end{aligned}
$$


which gives

$$
\begin{aligned}
0 \leq & \sum_{k=0}^{N} P_{k}+\sum_{k=0}^{N} 2\left(1-\delta_{k}^{(1)}\right) \alpha_{k}^{(1)}\left[-f_{1}\left(x_{k}^{(1)}, x^{*}\right)\right] \\
& +\sum_{k=0}^{N} 2\left(1-\delta_{k}^{(1)}\right) \alpha_{k}^{(2)}\left[-f_{2}\left(x_{k}^{(2)}, y^{*}\right)\right] \\
\leq & \left(\phi\left(x^{*}, x_{0}^{(1)}\right)+\phi\left(y^{*}, x_{0}^{(2)}\right)\right)-\left(\phi\left(x^{*}, x_{N+1}^{(1)}\right)+\phi\left(y^{*}, x_{N+1}^{(2)}\right)\right) \\
& +2 \sum_{k=0}^{N} \frac{\beta_{k}^{(1)} \varepsilon_{k}^{(1)}}{\rho_{k}^{(1)}}+2 \sum_{k=0}^{N} \frac{\left(\beta_{k}^{(1)}\right)^{2}}{\theta_{1}}+2 \sum_{k=0}^{N} \frac{\beta_{k}^{(2)} \varepsilon_{k}^{(2)}}{\rho_{k}^{(2)}}+2 \sum_{k=0}^{N} \frac{\left(\beta_{k}^{(2)}\right)^{2}}{\theta_{2}} .
\end{aligned}
$$

Letting $N \rightarrow \infty$, we have

$$
\begin{aligned}
0 \leq & \sum_{k=0}^{\infty} P_{k}+\sum_{k=0}^{\infty} 2\left(1-\delta_{k}^{(1)}\right) \alpha_{k}^{(1)}\left[-f_{1}\left(x_{k}^{(1)}, x^{*}\right)\right] \\
& +\sum_{k=0}^{\infty} 2\left(1-\delta_{k}^{(2)}\right) \alpha_{k}^{(2)}\left[-f_{2}\left(x_{k}^{(2)}, y^{*}\right)\right]<\infty
\end{aligned}
$$

Hence,

$$
\begin{gathered}
\sum_{k=0}^{\infty} P_{k}<\infty \\
\sum_{k=0}^{\infty} 2\left(1-\delta_{k}^{(1)}\right) \alpha_{k}^{(1)}\left[-f_{1}\left(x_{k}^{(1)}, x^{*}\right)\right]<\infty
\end{gathered}
$$

and

$$
\sum_{k=0}^{\infty} 2\left(1-\delta_{k}^{(2)}\right) \alpha_{k}^{(2)}\left[-f_{2}\left(x_{k}^{(2)}, y^{*}\right)\right]<\infty
$$

Since the sequence $\left\{x_{k}^{(1)}\right\}$ is bounded, then by the Condition B (B6) the sequence $\left\{w_{k}^{(1)}\right\}$ is also bounded. Thus there exists a real number $w^{(1)} \geq \rho^{(1)}$ such that $\left\|w_{k}^{(1)}\right\| \leq w^{(1)}$. Therefore, the conclusion follows as in Lemma 4.3 (ii) ((4.31)-(4.35)).

iii. From (4.51) and $0<a \leq \delta_{k}^{i} \leq b<1, i=1,2$, we have

$$
\lim _{k \rightarrow \infty}\left(D_{1}^{2}\left\|A_{1}^{*} J_{E_{3}}\left(A_{1} t_{k}^{(1)}-A_{2} t_{k}^{(2)}\right)\right\|^{2}+D_{2}^{2}\left\|A_{2}^{*} J_{E_{3}}\left(A_{1} t_{k}^{(1)}-A_{2} t_{k}^{(2)}\right)\right\|^{2}\right)=0 .
$$

Also $\lim _{k \rightarrow \infty} \phi\left(y_{k}^{(1)}, x_{k}^{(1)}\right)=0$, which implies $\lim _{k \rightarrow \infty}\left\|x_{k}^{(1)}-y_{k}^{(1)}\right\|=0$.

Similarly, $\lim _{k \rightarrow \infty} \phi\left(y_{k}^{(2)}, x_{k}^{(2)}\right)=0$, and consequently $\lim _{k \rightarrow \infty}\left\|x_{k}^{(2)}-y_{k}^{(2)}\right\|=0$. Now since $E_{1}$ is uniformly smooth, we have that the duality mapping $J_{E_{1}}$ is uniformly norm to norm continuous. From $\lim _{k \rightarrow \infty}\left\|x_{k}^{(1)}-y_{k}^{(1)}\right\|=0$, we have

$$
\begin{aligned}
\left\|J_{E_{(1)}} t_{k}^{(1)}-J_{E_{1}} x_{k}^{(1)}\right\| & =\left\|\delta_{k}^{(1)} J_{E_{1}} x_{k}^{(1)}+\left(1-\delta_{k}^{(1)}\right) J_{E_{1}} y_{k}^{(1)}-J_{E_{1}} x_{k}^{(1)}\right\| \\
& =\left(1-\delta_{k}^{(1)}\right)\left\|J_{E_{1}} y_{k}^{(1)}-J_{E_{1}} x_{k}^{(1)}\right\| .
\end{aligned}
$$

As $k \rightarrow \infty$, one has

$$
\left\|J_{E_{(1)}} t_{k}^{(1)}-J_{E_{1}} x_{k}^{(1)}\right\| \rightarrow 0
$$

Moreover, since $E_{1}$ is 2-uniformly convex, we have that $E_{1}^{*}$ is 2-uniformly smooth which implies it is uniformly smooth and thus $J_{E_{1}}^{-1}$ is uniformly norm to norm continuous. 
Therefore,

$$
\left\|t_{k}^{(1)}-x_{k}^{(1)}\right\|=\left\|J_{E_{1}}^{-1} J_{E_{1}} t_{k}^{(1)}-J_{E_{1}}^{-1} J_{E_{1}} x_{k}^{(1)}\right\| \rightarrow 0, k \rightarrow \infty .
$$

By the same line of argument, we have

$$
\left\|t_{k}^{(2)}-x_{k}^{(2)}\right\| \rightarrow 0, k \rightarrow \infty
$$

From (4.54), we have

$$
\lim _{k \rightarrow \infty}\left\|A_{1}^{*} J_{E_{3}}\left(A_{1} t_{k}^{(1)}-A_{2} t_{k}^{(2)}\right)\right\|^{2}=0
$$

and

$$
\lim _{k \rightarrow \infty}\left\|A_{2}^{*} J_{E_{3}}\left(A_{1} t_{k}^{(1)}-A_{2} t_{k}^{(2)}\right)\right\|^{2}=0 .
$$

Thus, from (4.41), (4.55) and (4.56), we have

$$
\begin{aligned}
\left\|A_{1} t_{k}^{(1)}-A_{2} t_{k}^{(2)}\right\|^{2} \leq & \left\|A_{1}^{*} J_{E_{3}}\left(A_{1} t_{k}^{(1)}-A_{2} t_{k}^{(1)}\right)\right\|\left\|t_{k}^{(1)}-x^{*}\right\| \\
& +\left\|A_{2}^{*} J_{E_{3}}\left(A_{1} t_{k}^{(1)}-A_{2} t_{k}^{(1)}\right)\right\|\left\|y^{*}-t_{k}^{(2)}\right\| \rightarrow 0 .
\end{aligned}
$$

Theorem 4.2. Assume that $f_{1}$ and $f_{2}$ satisfy condition $B$ and let $\left\{y_{k}^{(1)}\right\},\left\{y_{k}^{(2)}\right\},\left\{t_{k}^{(1)}\right\},\left\{t_{k}^{(2)}\right\}\left\{x_{k}^{(1)}\right\}$ and $\left\{x_{k}^{(2)}\right\}$ be the sequences generated by the Algorithm 1 and $\mu_{k}$ be as in Lemma 4.4. Then the sequences $\left\{\left(y_{k}^{(1)}, y_{k}^{(2)}\right)\right\},\left\{\left(t_{k}^{(1)}, t_{k}^{(2)}\right)\right\}$ and $\left\{\left(x_{k}^{(1)}, x_{k}^{(2)}\right)\right\}$ converge strongly to $(p, q) \in S$.

Proof. The proof is similar to the proof of Theorem 4.1 with Lemma 4.3 replaced with Lemma 4.5 and therefore it is omitted.

\section{Applications to the domain DeCOMposition FOR PDEs}

Let $E_{1}, E_{2}$ and $E_{3}$ be Banach spaces. Let $h_{1}: E_{1} \rightarrow \mathbb{R} \cup\{+\infty\}$ and $h_{2}: E_{2} \rightarrow \mathbb{R} \cup\{+\infty\}$ be two convex, lower semicontinuous and subdifferentiable functionals functionals. Let $A_{1}: E_{1} \rightarrow E_{3}$ and $A_{2}: E_{2} \rightarrow E_{3}$ be bounded linear operators. Let $f_{1}: E_{1} \times E_{1} \rightarrow \mathbb{R}$ and $f_{2}: E_{2} \times E_{2} \rightarrow \mathbb{R}$ be defined respectively as

$$
f_{1}(x, y):=h_{1}(y)-h_{1}(x)
$$

and

$$
f_{2}(x, y):=h_{2}(y)-h_{2}(x) .
$$

The SEEP (1.1)-(1.2) is reduced to the following split equality convex minimization problems: Find $x^{*} \in E_{1}, y^{*} \in E_{2}$ such that

$$
h_{1}\left(x^{*}\right) \leq h_{1}(x), \forall x \in E_{1} ; h_{2}\left(y^{*}\right) \leq h_{2}(y), \forall y \in E_{2},
$$

and

$$
A_{1} x^{*}=A_{2} y^{*}
$$

Equivalently, we have the following optimization problem with weak coupling in the constraint

$$
\min _{(x, y) \in E_{1} \times E_{2}}\left\{h_{1}(x)+h_{2}(y) ; A_{1} x=A_{2} y\right\} .
$$

Let us now convert the following problem arising from the domain decomposition for PDEs, (see [6]) to split equality convex minimization problems (5.1)-(5.2). 
Let $\Omega \subset \mathbb{R}^{n}$ be a bounded domain with $C^{2}$ boundary. Supposed that the set $\Omega$ is decomposed into two nonoverlapping Lipschitz subdomains $\Omega_{1}$ and $\Omega_{2}$ with a common interface $\Gamma$. Let $h \in L^{2}(\Omega)$ be a function and consider the following Neumann boundary value problem on $\Omega$

$$
\left\{\begin{array}{l}
-\Delta \omega=h \text { on } \Omega \\
\frac{\partial \omega}{\partial n}=0 \text { on } \partial \Omega
\end{array}\right.
$$

where $\frac{\partial \omega}{\partial n}=\nabla \omega \cdot \vec{n}$ and $\vec{n}$ is the unit outward normal to $\partial \Omega$. We make the assumption that $\int_{\Omega} h=0$, which is a necessary and sufficient condition for the existence of a solution. The weak solutions of the above Neumann problem satisfy the following minimization problem

$$
\min \left\{\frac{1}{2} \int_{\Omega}|\nabla \omega|^{2}-\int_{\Omega} h \omega ; \omega \in H^{1}(\Omega)\right\}
$$

see, for example, $[5,10]$. Furthermore, denoting by $\hat{\omega}$ a particular solution, the solution set of $(5.5)$ is of the form

$$
\{\hat{\omega}+k, k \in \mathbb{R}\}
$$

If $\Omega$ is of class $C^{2}$, we have from the regularity theory of weak solutions that $\hat{\omega} \in H^{2}(\Omega)$, see, for instance, $[1,20]$. Observe that, if $\omega \in H^{1}(\Omega)$, then the restrictions $u=\left.\omega\right|_{\Omega_{1}}$ and $v=\left.\omega\right|_{\Omega_{2}}$ belongs respectively to $H^{1}\left(\Omega_{1}\right)$ and $H^{1}\left(\Omega_{2}\right)$. Moreover $\left.u\right|_{\Gamma}=\left.v\right|_{\Gamma}$. Conversely, if $u \in H^{1}\left(\Omega_{1}\right), v \in H^{1}\left(\Omega_{2}\right)$ and $\left.u\right|_{\Gamma}=\left.v\right|_{\Gamma}$, then the function $\omega$ defined by

$$
\omega=\left\{\begin{array}{l}
u \text { on } \Omega_{1}, \\
v \text { on } \Omega_{2},
\end{array}\right.
$$

belongs to $H^{1}(\Omega)$. As a consequence, problem (5.5) can be reformulated as

$$
\min \left\{h_{1}(u)+h_{2}(u) ;(u, v) \in H^{1}\left(\Omega_{1}\right) \times H^{1}\left(\Omega_{2}\right) \text { and }\left.u\right|_{\Gamma}=\left.v\right|_{\Gamma}\right\}
$$

where

$$
h_{1}(u)=\frac{1}{2} \int_{\Omega_{1}}|\nabla u|^{2}-\int_{\Omega_{1}} h u
$$

and

$$
h_{2}(v)=\frac{1}{2} \int_{\Omega_{2}}|\nabla v|^{2}-\int_{\Omega_{2}} h v
$$

We can apply our Algorithm 1 to solve Problem (5.7) as follows: Let $E_{1}=H^{1}\left(\Omega_{1}\right), E_{2}=H^{1}\left(\Omega_{2}\right)$ and $E_{3}=L^{2}(\Gamma)$. Let the operators $A_{1}: E_{1} \rightarrow E_{3}$ and $A_{2}: E_{2} \rightarrow E_{3}$ be the trace operators on $\Gamma$, which are well-defined by the Lipschitz character of the boundaries of $\Omega_{1}$ and $\Omega_{2}$ (see ([8], Theorem 11.46) and ([21], Theorem 2])). Consequently, we propose the following method for solving Problem (5.7) (we take $\varepsilon_{k}^{(i)}=0$ for the sake of simplicity). 


\section{Algorithm 2}

1: Initialization: For each $i=1,2$, pick $x_{0}^{(i)} \in H^{1}\left(\Omega_{i}\right)$ and choose $\left\{\rho_{k}^{(i)}\right\},\left\{\beta_{k}^{(i)}\right\},\left\{\delta_{k}^{(i)}\right\},\left\{\varepsilon_{k}^{(i)}\right\}$ and $\left\{\mu_{k}\right\}$ such that $\rho_{k}^{(i)}>\rho^{(i)}>0, \beta_{k}^{(i)} \geq 0,0<a<\delta_{k}^{(i)}<b<1,0<\lambda \leq \mu_{k} \leq \gamma<\frac{2}{\left\|A_{1}\right\|_{H^{1}\left(\Omega_{1}\right)}^{2}+\left\|A_{2}\right\|_{H^{1}\left(\Omega_{2}\right)}^{2}}$, $\sum_{k=0}^{\infty} \frac{\beta_{k}^{(i)}}{\rho_{k}^{(i)}}=\infty$, and $\sum_{k=0}^{\infty}\left(\beta_{k}^{(i)}\right)^{2}<\infty$.

2: Find $w_{k}^{(i)} \in H^{1}\left(\Omega_{i}\right),(i=1,2)$ such that

$$
h_{i}(y) \geq h_{i}\left(x_{k}^{(i)}\right)+\left\langle w_{k}^{i}, y-x_{k}^{i}\right\rangle, \forall y \in H^{1}\left(\Omega_{i}\right) .
$$

Let $\eta_{k}^{(i)}=\max \left\{\rho_{k}^{(i)},\left\|w_{k}^{(i)}\right\|_{H^{1}\left(\Omega_{1}\right)}\right\}$ and $\alpha_{k}^{(i)}=\frac{\beta_{k}^{(i)}}{\eta_{k}^{(i)}}$.

3: Compute

$$
\left\{\begin{array}{l}
y_{k}^{(1)}=x_{k}^{(1)}-\alpha_{k}^{(1)} w_{k}^{(1)} \\
y_{k}^{(2)}=x_{k}^{(2)}-\alpha_{k}^{(2)} w_{k}^{(2)}
\end{array}\right.
$$

4: Compute

5: Compute

$$
\left\{\begin{array}{l}
t_{k}^{(1)}=\delta_{k}^{(1)} x_{k}^{(1)}+\left(1-\delta_{k}^{(1)}\right) y_{k}^{(1)} \\
t_{k}^{(2)}=\delta_{k}^{(2)} x_{k}^{(2)}+\left(1-\delta_{k}^{(2)}\right) y_{k}^{(2)}
\end{array}\right.
$$

$$
\left\{\begin{array}{l}
x_{k+1}^{(1)}=t_{k}^{(1)}-\mu_{k} A_{1}^{*}\left(A_{1} t_{k}^{(1)}-A_{2} t_{k}^{(2)}\right), \\
x_{k+1}^{(2)}=t_{k}^{(2)}+\mu_{k} A_{2}^{*}\left(A_{1} t_{k}^{(1)}-A_{2} t_{k}^{(2)}\right) .
\end{array}\right.
$$

6: Set $\mathrm{k}:=\mathrm{k}+1$ and go to 2 .

Theorem 5.1. Let $\Omega \subset \mathbb{R}^{N}$ be a bounded domain which can be decomposed in two nonoverlapping Lipschitz subdomains $\Omega_{1}$ and $\Omega_{2}$ with a common interface $\Gamma$. We assume that $\Omega$ is of class $C^{2}$. Let $h \in L^{2}(\Omega)$ be such that $\int_{\Omega} h=0$ and let the functions $h_{1}: H^{1}\left(\Omega_{1}\right) \rightarrow \mathbb{R} \cup\{+\infty\}$ and $h_{2}: H^{1}\left(\Omega_{2}\right) \rightarrow$ $\mathbb{R} \cup\{+\infty\}$ be as defined above. Let $\left\{y_{k}^{(1)}\right\},\left\{y_{k}^{(2)}\right\},\left\{t_{k}^{(1)}\right\},\left\{t_{k}^{(2)}\right\}\left\{x_{k}^{(1)}\right\}$ and $x_{k}^{(2)}$ be the sequences generated by the Algorithm 2. Then the sequences $\left\{\left(y_{k}^{(1)}, y_{k}^{(2)}\right)\right\},\left\{\left(t_{k}^{(1)}, t_{k}^{(2)}\right)\right\}$ and $\left\{\left(x_{k}^{(1)}, x_{k}^{(2)}\right)\right\}$ converge strongly to $(\hat{u}, \hat{v}) \in H^{1}\left(\Omega_{1}\right) \times H^{1}\left(\Omega_{2}\right)$, where $(\hat{u}, \hat{v})$ is such that the map

$$
\hat{\omega}=\left\{\begin{array}{l}
\hat{u} \text { on } \Omega_{1}, \\
\hat{v} \text { on } \Omega_{2},
\end{array}\right.
$$

is a solution of the Neumann problem (5.4).

\section{Acknowledgments}

The first author was supported by the National Research Foundation (NRF) of South Africa (Grant Numbers: 111992). Opinions expressed and conclusions arrived are those of the authors and are not necessarily to be attributed to the NRF. The research of the second author was supported by the Alexander von Humboldt-Foundation. The authors are grateful to the reviewers for useful suggestions which improved the contents of this paper.

\section{REFERENCES}

[1] S. Agmon, A. Douglis, L. Nirenberg, Estimates near the boundary for solutions of elliptic partial differential equations satisfying general boundary conditions I, Comm. Pure Appl. Math. 12 (1959), 623-727. 
[2] Y.I. Alber, Metric and generalized projection operators in Banach spaces: Properties and applications, in: A.G. Kartsatos (Ed.), Theory and Applications of Nonlinear Operators of Accretive and Monotone Type, in: Lecture Notes Pure Appl. Math., vol. 178, Dekker, New York, 1996, pp. 15-50.

[3] Y.I. Alber, S. Reich, An iterative method for solving a class of nonlinear operator equations in Banach spaces, PanAmer. Math. J. 4 (1994), 39-54.

[4] K. Aoyama, F. Kohsaka, Strongly relatively nonexpansive sequences generated by firmly nonexpansive-like mappings, Fixed Point Theory Appl. 2014 (2-14), Article ID 95.

[5] H. Attouch, G. Buttazzo, G. Michaille, Variational analysis in Sobolev and BV spaces, Applications to PDEs and optimization, in: MPS/SIAM Series on Optimization, vol. 6, Society for Industrial and Applied Mathematics (SIAM), Philadelphia, PA, 2006.

[6] H. Attouch, A. Cabot, P. Frankel, J. Peypouquet, Alternating proximal algorithms for linearly constrained variational inequalities: Application to domain decomposition for PDEs, Nonlinear Anal. 74 (2011), 7455-7473

[7] K. Avetisyan, O. Djordjevic, M. Pavlovic, Littlewood-Paley inequalities in uniformly convex and uniformly smooth Banach spaces, J. Math. Anal. Appl. 336 (2007), 31-43.

[8] D. Azé, Eléments d'Analyse Convexe et Variationnelle, Ellipses, Paris, 1997.

[9] E. Blum, W. Oettli, From optimization and variational inequalities to equilibrium problems, Math. Student 63 (1994), 123-145.

[10] H. Brézis, Analyse Fonctionnelle: Théorie et Applications, Dunod, Paris, 1999.

[11] Y. Censor, T. Bortfeld, B. Martin, A. Trofimov, A unified approach for inversion problem in intensity-modulated radiation therapy, Phys. Med. Biol. 51 (2006), 2353-2365.

[12] Y. Censor, T. Elfving, A multiprojection algorithm using Bregman projections in a product space., Numer. Algo. 8 (1994), 221-329.

[13] Y. Censor, T. Elfving, N. Kopf, T. Bortfeld, The multiple-sets split feasibility problem and its applications for inverse problems, Inverse Probl. 21 (2005), 2071-2084.

[14] Y. Censor, A. Gibali, S. Reich, Algorithm for the split variational inequality problem, Numer. Algo. 59 (2012), $301-323$.

[15] Y. Censor, A. Segal, The split common fixed point problem for directed operators, J. Convex Anal. 16 (2009), 587-600.

[16] C.E. Chidume, U.V. Nnyaba, O.M. Romanus, A new algorithm for variational inequality problems with a generalized phi-strongly monotone map over the set of common fixed points of a finite family of quasi-phi-nonexpansive maps, with applications, J. Fixed Point Theory Appl. 20 (2018), Article ID 29.

[17] C.E. Chidume, O.M. Romanus, U.V. Nnyaba, An iterative algorithm for solving split equilibrium problems and split equality variational inclusions for a class of nonexpansive-type maps, Optimization 67 (2018), 1949-1962.

[18] M. Eslamain, Strong convergence of split equality variational inequality and fixed point problem, Riv. Math. Univ. Parma (N.S.) 8 (2017), 225-246.

[19] A. G. Gebrie, R. Wangkeeree, Hybrid projected subgradient-proximal algorithms for solving split equilibrium problems and split common fixed point problems of nonexpansive mappings in Hilbert spaces, Fixed Point Theory Appl. 2018 (2018), Article ID 5.

[20] D. Gilbarg, N. Trudinger, Elliptic Partial Differential Equations of Second Order, Springer-Verlag, Berlin, 1977.

[21] J. Marschall, The trace of Sobolev-Slobodeckij spaces on Lipschitz domains, Manuscripta Math. 58 (1987), 47-65.

[22] S. Matsushita, W. Takahashi, A strong convergence theorem for relatively nonexpansive mappings in a Banach space, J. Approx. Theory 134 (2005), 257-266.

[23] L.D. Muu, W. Oettli, Convergence of an adaptive penalty scheme for finding constrained equilibria, Nonlinear Anal. 18 (1992), 1159-1166.

[24] K. Nakajo, Strong convergence for gradient projection method and relatively nonexpansive mappings in Banach spaces, Appl. Math. Comput. 271 (2015), 251-258.

[25] F.U. Ogbuisi, O.T. Mewomo, On split generalised mixed equilibrium problems and fixed-point problems with no prior knowledge of operator norm, J. Fixed Point Theory Appl. 19 (2017), 2109-2128.

[26] F.U. Ogbuisi, An iterative method for solving split generalized mixed equilibrium and fixed point problems in Banach spaces, J. Nonlinear Convex Anal. 19 (2018), 803-821. 
[27] X. Qin, Y.J. Cho, S.M. Kang, Convergence theorems of common elements for equilibrium problems and fixed point problems in Banach spaces, J. Comput. Appl. Math. 225 (2009), 20-30.

[28] Y. Shehu, Strong convergence theorem for multiple sets split feasibility problems in Banach spaces, Numer. Funct. Anal. Optim. 37 (2016), 1021-1036.

[29] Y. Shehu, O.T. Mewomo, F.U. Ogbuisi, Further investigation into approximation of a common solution of fixed point problems and split feasibility problems, Acta Math. Sci. Ser. B (Engl. Ed.) 36 (2016), 913-930.

[30] Y. Shehu, F.U. Ogbuisi, O.S. Iyiola, Convergence analysis of an iterative algorithm for fixed point problems and split feasibility problems in certain Banach spaces, Optimization 65 (2016), 299-323.

[31] R. Shukla, R. Pant, Approximating solution of split equality and equilibrium problems by viscosity approximation algorithms, Comput. Appl. Math. 37 (2018), 5293-5314.

[32] Y. Takahashi, K. Hashimoto, M. Kato, On sharp uniform convexity, smoothness, and strong type, cotype inequalities, J. Nonlinear Convex Anal. 3 (2002), 267-281.

[33] S. Takahashi, W. Takahashi, Viscosity approximation methods for equilibrium problems and fixed point problems in Hilbert spaces, J. Math. Anal. Appl. 331 (2007), 506-515.

[34] H.K. Xu, Inequalities in Banach spaces with applications, Nonlinear Anal. 16 (1991), 1127-1138. 